\title{
EXTREMAL MAPPINGS OF FINITE DISTORTION
}

\author{
K. ASTALA, T. IWANIEC, G. J. MARTIN AND J. ONNINEN
}

\section{Introduction}

The theory of mappings of finite distortion has arisen out of a need to extend the ideas and applications of the classical theory of quasiconformal mappings to the degenerate elliptic setting. There one finds concrete applications in materials science, particularly non-linear elasticity and critical phase phenomena, and the calculus of variations.

In this paper we refine and extend these connections by initiating the study of extremal problems for mappings with finite distortion.

There are many natural reasons for studying such problems. First, we eventually hope to lay down the analytical foundations for approaches to compactifying the moduli spaces for holomorphic dynamical systems such as Teichmüller spaces where it is our expectation that a compactification will be by mappings of finite distortion whose distortion function lies in some natural integrability class.

Secondly, we find that there are many new and unexpected phenomena concerning existence, uniqueness and regularity for these extremal problems where the functionals are polyconvex but typically not convex. These seem to differ markedly from phenomena observed when studying multi-well type functionals.

Thus our primary aim here is to extend the theory of extremal quasiconformal mappings by considering integral averages of the distortion function instead of its $\mathcal{L}^{\infty}$-norm. Let us indicate the sorts of results we shall prove here by a surprising example in two dimensions. Suppose $f_{o}: \mathbb{S} \rightarrow \mathbb{S}$ is a homeomorphism of the circle. We ask what is the mapping $f: \mathbb{D} \rightarrow \mathbb{D}$ of the disk with $f \mid \mathbb{S}=f_{o}$, whose distortion function is minimal in $\mathcal{L}^{1}$-norm. We show that if $f_{o}^{-1} \in \mathcal{W}^{1 / 2,2}(\mathbb{S})$, then there is a unique extremal which is a real analytic diffeomorphism with non-vanishing Jacobian determinant. The condition on $f_{o}$ is sharp. Contrast this with the classical theory [31] where the mapping $f_{o}$ must be assumed quasisymmetric (a much stronger assumption). Then there is an extremal quasiconformal mapping with boundary values $f_{o}$, but it is not always unique and it is seldom smooth. Indeed, even when $f_{o}$ is quasisymmetric, the $\mathcal{L}^{1}$-minimiser for the distortion function will almost never be quasiconformal. This result is doubly surprising when we note that there is no general modulus of continuity (so no compactness) nor general improved regularity theory for mappings with only integrable distortion.

Received 1 June 2004; revised 2 February 2005.

2000 Mathematics Subject Classification 30C60, 35J15, 35J70.

Astala was supported in part by the Academy of Finland, projects 34082 and 41933, Iwaniec by the National Science Foundation grants DMS-0301582 and DMS-0244297, Martin by the N.Z. Marsden Fund and the N.Z. Royal Society (James Cook Fellowship), and Onninen by the National Science Foundation grant DMS-0400611. 
Away from the $\mathcal{L}^{1}$-theory and closer to the $\mathcal{L}^{\infty}$-theory we have mappings whose distortion function is exponentially integrable. In this case there are substantial results concerning modulus of continuity and regularity available, $[\mathbf{1 6}]$. From these one obtains existence of minimisers and some improved regularity for the boundary value problem as discussed above. However establishing uniqueness seems difficult.

There are also other natural problems that we shall consider such as the generalizations to the $\mathcal{L}^{1}$ setting of the famous Grötzsch problem concerning extremal mappings between $n$-dimensional boxes; see $\S \S 6$ and 7 .

Finally, we wish to make an observation. The principal advantage of minimising over families of homeomorphisms in the above variational problems lies in the fact that the inverse maps are also extremal for their own variational integrals. Sometimes this associated problem is easier to solve than the original one as it may involve minimising a convex functional. For instance, in $n$-dimensions, the $\mathcal{L}^{1}$ minimisation problem leads to the $n$-harmonic equation for the inverse of an extremal mapping.

\section{Formulation of the general problem}

We begin by defining the class of mappings which will most concern us here. A mapping $f: \Omega \rightarrow \Omega^{\prime}$ between subdomains of $\mathbb{R}^{n}$ and of Sobolev class $\mathcal{W}_{\text {loc }}^{1,1}\left(\Omega, \Omega^{\prime}\right)$ is said to have finite distortion if there is a measurable function $1 \leqslant K(x)<\infty$ such that

$$
|D f(x)|^{n} \leqslant K(x) J(x, f)
$$

and if $J(z, f) \in L_{\mathrm{loc}}^{1}(\Omega)$. Here $|D f(x)|$ is the operator norm,

$$
|D f|=\max \{|D f v|:|v|=1\},
$$

of the linear differential map $D f(x)$ and $J(x, f)=\operatorname{det} D f(x)$ is its Jacobian determinant. The reader may observe that for homeomorphisms the condition $J(z, f) \in L_{\text {loc }}^{1}(\Omega)$ is largely redundant. The smallest function $K(x)$ for which the distortion inequality (2.1) holds is called the outer distortion of $f$, defined by

$$
K(x, f)=\frac{|D f(x)|^{n}}{J(x, f)}
$$

at points where $D f(x)$ exists and is non-singular and we set $K(x, f)=1$ elsewhere. The operator norm of the differential matrix at (2.2) has the disadvantage of being insufficiently regular to deal with variational equations. We therefore introduce the outer distortion function

$$
\mathbb{K}(x, f)=\frac{\|D f(x)\|^{n}}{J(x, f)}
$$

at points where $D f(x)$ exists and is non-singular and set $\mathbb{K}(x, f)=1$ elsewhere. Here $\|A\|^{2}=n^{-1} \operatorname{tr}\left(A^{t} A\right)$ is the mean Hilbert-Schmidt norm. Notice that in two dimensions

$$
\mathbb{K}(x, f)=\frac{1}{2}\left(K(x, f)+\frac{1}{K(x, f)}\right) \quad(n=2)
$$


is a convex function of $K(x, f)$ and therefore

$$
\|\mathbb{K}(x, f)\|_{\infty}=\frac{1}{2}\left(\|K(x, f)\|_{\infty}+\frac{1}{\|K(x, f)\|_{\infty}}\right) \quad(n=2),
$$

so that minimising either $\|\mathbb{K}(x, f)\|_{\infty}$ or $\|K(x, f)\|_{\infty}$ leads to the same quasiconformal minimisers.

More generally, to every strictly increasing convex function $\Psi:[1, \infty) \rightarrow[1, \infty)$, with $\Psi(1)=1$, we associate two further distortion functions

$$
K_{\Psi}(x, f)=\Psi(K(x, f)) \quad \text { and } \quad \mathbb{K}_{\Psi}(x, f)=\Psi(\mathbb{K}(x, f)) .
$$

We can now formulate the major minimisation problem that we shall address. Let $\mathcal{F}$ consist of homeomorphisms $f: \Omega \rightarrow \Omega^{\prime}$ of finite distortion such that

$$
\int_{\Omega} \mathbb{K}_{\Psi}(x, f) d x<\infty
$$

Problem 1. Given $f_{o} \in \mathcal{F}$ find a mapping $f \in \mathcal{F}$ which coincides with $f_{o}$ on $\partial \Omega$ and minimizes the integral at (2.6).

We shall also consider related problems where we do not prescribe the precise boundary values or where we minimise integral averages of other distortion functions. We now give a fuller discussion of these.

\section{Distortion functions}

Distortion functions are designed to measure the deviation from conformality of a given mapping $f: \Omega \rightarrow \Omega^{\prime}$ by considering the linear differential map $D f(x): \mathbb{R}^{n} \rightarrow \mathbb{R}^{n}$. We shall denote the set of all $n \times n$-matrices by $\mathbb{R}^{n \times n}$, and those with positive determinant by $\mathbb{R}_{+}^{n \times n}$. It will be convenient to include the zero matrix in the domain of distortion functions. The conformal matrices are

$$
\mathrm{CO}_{+}(n)=\left\{A:|A|^{n}=\operatorname{det} A\right\}
$$

equivalently the defining equation could be $\|A\|^{n}=\operatorname{det} A$. In two dimensions it is advantageous to use complex variables. A general linear transformation takes the form

$$
A z=a z+b \bar{z} \quad \text { where } a, b \in \mathbb{C} .
$$

The determinant, the operator norm and mean Hilbert-Schmidt norm are then

$$
\operatorname{det} A=|a|^{2}-|b|^{2}, \quad|A|=|a|+|b|, \quad\|A\|^{2}=|a|^{2}+|b|^{2} .
$$

To every pair of ordered $\ell$-tuples $I=\left(i_{1}, \ldots, i_{\ell}\right)$ and $J=\left(j_{1}, \ldots, j_{\ell}\right)$, with $1 \leqslant i_{1}<\ldots<i_{\ell} \leqslant n$ and $1 \leqslant j_{1}<\ldots<j_{\ell} \leqslant n$, there corresponds the $\ell \times \ell$-subdeterminant of a matrix $A=\left[A_{j}^{i}\right] \in \mathbb{R}^{n \times n}$, namely

$$
A_{J}^{I}=\operatorname{det}\left[\begin{array}{ccc}
A_{j_{1}}^{i_{1}} & \cdots & A_{j_{\ell}}^{i_{1}} \\
\vdots & & \vdots \\
A_{j_{1}}^{i_{\ell}} & \cdots & A_{j_{\ell}}^{i_{\ell}}
\end{array}\right] .
$$


The $\ell$ th exterior power of $A$ is the $\left(\begin{array}{l}n \\ \ell\end{array}\right) \times\left(\begin{array}{l}n \\ \ell\end{array}\right)$-matrix

$$
A^{\ell \times \ell}=\left[A_{J}^{I}\right] \in \mathbb{R}^{\left(\begin{array}{l}
n \\
\ell
\end{array}\right) \times\left(\begin{array}{l}
n \\
\ell
\end{array}\right)}
$$

indexed by $\ell$-tuples $I=\left(i_{1}, \ldots, i_{\ell}\right)$ and $J=\left(j_{1}, \ldots, j_{\ell}\right)$. We shall need the following well-known identities from exterior algebra:

$$
\begin{aligned}
{[A B]^{\ell \times \ell} } & =A^{\ell \times \ell} B^{\ell \times \ell}, \quad\left[A^{t}\right]^{\ell \times \ell}=\left[A^{\ell \times \ell}\right]^{t}, \\
{\left[A^{-1}\right]^{\ell \times \ell} } & =\left[A^{\ell \times \ell}\right]^{-1}, \quad \operatorname{det} A^{\ell \times \ell}=(\operatorname{det} A)^{\ell} .
\end{aligned}
$$

Then Cramer's rule states

$$
A^{t} A_{\sharp}=(\operatorname{det} A) \mathbf{I}
$$

where $A_{\sharp}$ is the cofactor matrix. To every pair $(i, j)$ there corresponds the $(i, j)$ cofactor, which is the product of $(-1)^{i+j}$ with the $(n-1) \times(n-1)$ subdeterminant obtained by deleting the $i$ th row and the $j$ th column in $A$. Cramer's rule can

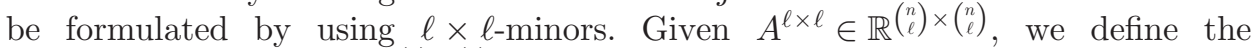

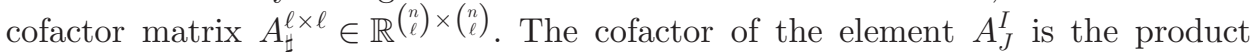
of $(-1)^{i_{1}+\ldots+i_{\ell}+j_{1}+\ldots+j_{\ell}}$ with the $(n-\ell) \times(n-\ell)$-subdeterminant obtained by suppressing the $i_{1}, \ldots, i_{\ell}$ th rows and $j_{1}, \ldots, j_{\ell}$ th columns. Cramer's rule reads as

$$
\left[A^{\ell \times \ell}\right]^{t} A_{\sharp}^{\ell \times \ell}=(\operatorname{det} A) \mathbf{I} .
$$

Thus in particular, if $\operatorname{det} A \neq 0$, then

$$
\left[A_{\sharp}^{\ell \times \ell}\right]^{t}=(\operatorname{det} A)\left[A^{-1}\right]^{\ell \times \ell} .
$$

There is an isometry, called the Hodge star operator, which assigns to $A_{\sharp}^{\ell \times \ell}$ the $(n-\ell)$-exterior power of $A$. This isometry arises from identifying the $\ell$-tuple $I=$ $\left(i_{1}, \ldots, i_{\ell}\right)$ with its complementary $(n-\ell)$-tuple. Hence

$$
\left|A_{\sharp}^{\ell \times \ell}\right|=\left|A^{(n-\ell) \times(n-\ell)}\right| \quad \text { and } \quad\left\|A_{\sharp}^{\ell \times \ell}\right\|=\left\|A^{(n-\ell) \times(n-\ell)}\right\| .
$$

These identities be can easily derived by reducing $A$ to a diagonal matrix.

The following distortion functions will interest us most as they have the very important property of being polyconvex; see Appendix A.2. For each $A \in \mathbb{R}_{+}^{n \times n}$ and $\ell=1,2, \ldots, n-1$, we define

$$
K_{\ell}(A)=\frac{\left|A^{\ell \times \ell}\right|^{n /(n-\ell)}}{(\operatorname{det} A)^{\ell /(n-\ell)}}, \quad \mathbb{K}_{\ell}(A)=\frac{\left\|A^{\ell \times \ell}\right\|^{n /(n-\ell)}}{(\operatorname{det} A)^{\ell /(n-\ell)}} .
$$

We extend this definition to $\mathbb{R}_{+}^{n \times n} \cup\{0\}$ by setting $K_{\ell}(0)=\mathbb{K}_{\ell}(0)=1$. Notice that $K_{\ell}(A) \geqslant 1$ and $\mathbb{K}_{\ell}(A) \geqslant 1$, with equality occurring if and only if $A \in \mathrm{CO}_{+}(n)$.

The two outer distortion functions discussed previously in (2.2) and (2.3) arise when $\ell=1$ as

$$
K(A)=K_{1}(A)^{n-1} \text { and } \quad \mathbb{K}(A)=\mathbb{K}_{1}(A)^{n-1} .
$$

The corresponding inner distortion functions are

$$
K_{I}(A)=K_{n-1}(A)=K\left(A^{-1}\right), \quad \mathbb{K}_{I}(A)=\mathbb{K}_{n-1}(A)=\mathbb{K}\left(A^{-1}\right) .
$$

When $n=2$ the inner distortions are the same as the outer distortions. 
Given the above, a mapping $f \in \mathcal{W}_{\text {loc }}^{1,1}\left(\Omega, \Omega^{\prime}\right)$ will have finite distortion if $D f(x) \in \mathbb{R}_{+}^{n \times n} \cup\{0\}$ for almost every $x \in \Omega$ and $J(x, f)=\operatorname{det} D f \in \mathcal{L}_{\text {loc }}^{1}(\Omega)$. Having defined distortion functions for matrices in $\mathbb{R}_{+}^{n \times n} \cup\{0\}$ we define for mappings $f: \Omega \rightarrow \Omega^{\prime}$ of finite distortion:

$$
\begin{aligned}
& K_{\ell}(x, f)=K_{\ell}(D f(x))=\frac{\left|D^{\ell \times \ell} f(x)\right|^{n /(n-\ell)}}{J(x, f)^{\ell /(n-\ell)}}, \\
& \mathbb{K}_{\ell}(x, f)=\mathbb{K}_{\ell}(D f(x))=\frac{\left\|D^{\ell \times \ell} f(x)\right\|^{n /(n-\ell)}}{J(x, f)^{\ell /(n-\ell)}} .
\end{aligned}
$$

The reader may find more about distortion functions in Appendix A.1.

\section{Variations of weakly differentiable homeomorphisms}

As this paper is largely concerned with Sobolev classes of homeomorphisms we shall need to establish the existence of suitable variations of such mappings. These variations may be required to preserve quasiconformality as well as some other natural properties of mappings. The following theorem captures all those needs.

Theorem 4.1. Let $f: \mathbb{B} \rightarrow \mathbb{R}^{n}$ be a homeomorphism onto its image of the Sobolev class $\mathcal{W}_{\text {loc }}^{1,1}\left(\mathbb{B}, \mathbb{R}^{n}\right)$ and let $a \in \mathbb{B}$ be a Lebesgue point of $D f$ such that $J(a, f)>0$. Then there exists a diffeomorphism $h: \mathbb{B} \rightarrow \mathbb{B}$, referred to as change of variables, such that the composite mapping $\widetilde{f}(x)=f(h(x))$ satisfies:

(i) $\tilde{f}(x)=f(x)$ near $\partial \mathbb{B}$;

(ii) the origin is a Lebesgue point of $D \tilde{f}$;

(iii) $D \widetilde{f}(0)=\mathbf{I}$.

The proof for the construction of this change of variables consists of three lemmas that are of independent interest.

Lemma 4.1. Given a point $a \in \mathbb{B}$ and $r>|a|$ there exists a diffeomorphism $\Phi: \mathbb{R}^{n} \rightarrow \mathbb{R}^{n}$ such that

$$
\Phi(0)=a, \quad D \Phi(0)=\mathbf{I}, \quad \text { and } \quad \Phi(x)=x \text { for }|x| \geqslant r .
$$

Proof. Such a diffeomorphism $\Phi$ will be a perturbation of the identity map, namely

$$
\Phi(x)=x+\eta(x) a
$$

where $\eta \in \mathcal{C}_{0}^{\infty}\left(\mathbb{B}_{r}\right)$ satisfies

$$
0 \leqslant \eta \leqslant 1, \quad \eta(0)=1, \quad \text { and } \quad\|\nabla \eta\|_{\infty}<\frac{1}{|a|} .
$$

Its differential is a matrix of the form

$$
D \Phi(x)=\mathbf{I}+a \otimes \nabla \eta .
$$

Since $\eta$ assumes its largest value 1 at the origin, we infer that $\nabla \eta(0)=0$. Hence $D \Phi(0)=\mathbf{I}$, as claimed. The Jacobian determinant of $\Phi$ is computed in $\mathbb{B}_{r}$ as

$$
\operatorname{det} D \Phi=1+\langle a, \nabla \eta\rangle \geqslant 1-|a||\nabla \eta| \geqslant 1-|a||| \nabla \eta \|_{\infty}>0
$$


and $\operatorname{det} D \Phi=1$ outside $\mathbb{B}_{r}$. In particular, $\Phi$ is a local diffeomorphism of $\mathbb{R}^{n}$ into itself. Since $\Phi(x)=x$ outside the ball $\mathbb{B}_{r}$, by topological arguments we conclude that $\Phi: \mathbb{R}^{n} \rightarrow \mathbb{R}^{n}$ is one-to-one.

Lemma 4.2 (Decomposition of matrices). Given $A \in \mathbb{R}_{+}^{n \times n}$ and $\epsilon>0$, there exist $A_{1}, A_{2}, \ldots, A_{k} \in \mathbb{R}_{+}^{n \times n}$ such that

$$
\left|\mathbf{I}-A_{i}\right| \leqslant \epsilon \quad \text { for } i=1,2, \ldots, k
$$

and

$$
A=A_{1} \cdot A_{2} \cdot \ldots \cdot A_{k}
$$

Proof. As $\mathbb{R}_{+}^{n \times n}$ is a connected matrix Lie group, we can decompose $A$ as

$$
A=e^{X_{1}} e^{X_{2}} \cdot \ldots \cdot e^{X_{m}}
$$

where $X_{1}, X_{2}, \ldots, X_{m} \in \mathbb{R}^{n \times n}$; see [11, Corollary 2.31]. Next choose $\delta=N^{-1}$, where $N$ is a large positive integer such that $e^{\delta\left|X_{k}\right|}-1 \leqslant \epsilon$ for $k=1,2, \ldots, m$. Hence

$$
\begin{aligned}
A & =\underbrace{e^{\delta X_{1}} \ldots e^{\delta X_{1}}}_{N \text { times }} \cdot \underbrace{e^{\delta X_{2}} \ldots e^{\delta X_{2}}}_{N \text { times }} \cdot \ldots \cdot \underbrace{e^{\delta X_{m}} \ldots e^{\delta X_{m}}}_{N \text { times }} \\
& =: A_{1} \cdot A_{2} \cdot \ldots \cdot A_{k}, \quad \text { where } k=m N .
\end{aligned}
$$

For every $A_{i}$ we have

$$
\left|\mathbf{I}-A_{i}\right| \leqslant \max _{1 \leqslant k \leqslant m}\left|1-e^{\delta X_{k}}\right| \leqslant \max _{1 \leqslant k \leqslant m}\left|1-e^{\delta\left|X_{k}\right|}\right| \leqslant \epsilon,
$$

as desired.

LEMma 4.3. Given $A \in \mathbb{R}_{+}^{n \times n}$ and $r>0$, there exists a diffeomorphism $\Psi: \mathbb{R}^{n} \rightarrow \mathbb{R}^{n}$ such that

$$
\Psi(0)=0, \quad D \Psi(0)=A, \quad \text { and } \quad \Psi(x)=x \text { for }|x| \geqslant r .
$$

Proof. First assume that $A$ is sufficiently close to the identity matrix, say

$$
|\mathbf{I}-A|<\frac{1}{4} \text {. }
$$

We construct $\Psi$ as a perturbation of the identity $\Psi(x)=x-(x-A x) \eta$ where $\eta \in \mathcal{C}_{0}^{\infty}\left(\mathbb{B}_{r}\right), 0 \leqslant \eta(x) \leqslant 1, \eta(0)=1$, and $\|\nabla \eta\|_{\infty}<2 / r$. We find the differential of $\Psi$ as follows:

$$
D \Psi(x)=\mathbf{I}-(\mathbf{I}-A) \eta-(x-A x) \otimes \nabla \eta .
$$

Clearly, $D \Psi(0)=A$. In order to see that $\operatorname{det} D \Psi(x) \neq 0$, we view $D \Psi(x)$ as a small perturbation of $\mathbf{I}$. For $|x| \leqslant r$ we have the following estimate of the perturbation term:

$$
\begin{aligned}
|\eta(x)(\mathbf{I}-A)+(x-A x) \otimes \nabla \eta| & \leqslant \eta(x)|\mathbf{I}-A|+|x-A x||\nabla \eta(x)| \\
& \leqslant|\mathbf{I}-A|+r|\mathbf{I}-A|\|\nabla \eta\|_{\infty} \\
& \leqslant 3|\mathbf{I}-A|<\frac{3}{4} .
\end{aligned}
$$

It follows that $\operatorname{det} D \Psi(x)>4^{-n}$. As in Lemma 4.1 we conclude that $\Psi$ is a diffeomorphism of $\mathbb{R}^{n}$ onto itself. We now can free ourselves from the assumption 
(4.10) by using the decomposition of Lemma 4.2. Accordingly we put $A=$ $A_{1} \cdot A_{2} \cdot \ldots \cdot A_{k}$ where $\left|\mathbf{I}-A_{i}\right|<\frac{1}{4}$ for $i=1,2, \ldots, k$. Let $\Psi_{1}, \Psi_{2}, \ldots, \Psi_{k}: \mathbb{R}^{n} \rightarrow \mathbb{R}^{n}$ be the diffeomorphisms constructed above, that is,

$$
\Psi_{i}(0)=0, \quad \Psi_{i}(x)=x \text { for }|x| \geqslant r, \quad \text { and } \quad D \Psi_{i}(0)=A_{i} \text { for } i=1,2, \ldots, k .
$$

The composition $\Psi=\Psi_{1} \circ \Psi_{2} \circ \ldots \circ \Psi_{k}: \mathbb{R}^{n} \rightarrow \mathbb{R}^{n}$ satisfies all the assertions of Lemma 4.3.

Proof of Theorem 4.1. We define $h$ as the composition of $\Phi$ from Lemma 4.1 and $\Psi$ from Lemma 4.3. This latter map $\Psi$ is determined by taking $A=[D f(a)]^{-1} \in \mathbb{R}_{+}^{n \times n}$. Actually, the composite map

$$
h=\Phi \circ \Psi: \mathbb{R}^{n} \rightarrow \mathbb{R}^{n}, \quad h(0)=a,
$$

is a diffeomorphism of the entire space $\mathbb{R}^{n}$. It maps the unit ball onto itself, and is the identity near $\partial \mathbb{B}$. The chain rule applies to $\widetilde{f}$ as follows:

$$
D \tilde{f}(x)=D f(h(x)) D f(x) \text { for almost every } x \in \mathbb{B} \text {. }
$$

In particular, the origin is a Lebesgue point of $D \widetilde{f}$. Moreover,

$$
D \widetilde{f}(0)=D f(a) D h(0)=\mathbf{I}
$$

because

$$
D h(0)=D \Phi(0) D \Psi(0)=D \Psi(0)=[D f(a)]^{-1}
$$

\section{Sublinear growth, the failure of minimization}

We shall henceforth use the mean value notation

$$
f_{\Omega} f=\frac{1}{|\Omega|} \iint_{\Omega} f
$$

Given a mapping $f_{o}: \overline{\mathbb{B}} \rightarrow \mathbb{R}^{n}$ we now study minima of the variational integral

$$
\min \int_{\mathbb{B}} \Psi\left[K_{I}(x, f)\right] d x
$$

in the class $\mathcal{F}_{o}=\mathcal{F}\left(f_{o}\right)$ of all $\mathcal{W}^{1, n}\left(\mathbb{B}, \mathbb{R}^{n}\right)$ homeomorphisms $f: \overline{\mathbb{B}} \rightarrow \mathbb{R}^{n}$ which coincide with $f_{o}$ on $\partial \mathbb{B}$. We demonstrate that these integrals may not attain the minimum value if $\Psi$ exhibits sublinear growth, meaning that

$$
\lim _{t \rightarrow \infty} \frac{\Psi(t)}{t}=0
$$

In many ways this situation is reminiscent of the well-known Lavrentiev phenomenon in the Calculus of Variations [21]. For simplicity we consider quasiconformal boundary data. The reader may easily generalise this situation.

Theorem 5.3. Let $\Psi \in \mathcal{C}[1, \infty)$ be a positive strictly increasing function of sublinear growth. Given a quasiconformal map $f_{o}: \overline{\mathbb{B}} \rightarrow \mathbb{R}^{n}$ we have

$$
\inf _{f \in \mathcal{F}_{o}} f_{\mathbb{B}} \Psi\left[K_{I}(x, f)\right] d x=\Psi(1)
$$


In particular, the minimization problem (5.1) has no solution in $\mathcal{F}_{o}$ if $f_{o}: \partial \mathbb{B} \rightarrow \mathbb{R}^{n}$ admits no conformal extension to $\mathbb{B}$.

Proof. Let $a \in \mathbb{B}$ be a Lebesgue point for both $D f(x)$ and $\Psi\left[K_{I}\left(x, f_{o}\right)\right]$ such that $\operatorname{det} D f_{o}(a)>0$. We shall make use of Theorem 4.1 to modify $f_{o}$ without changing its boundary values and quasiconformality. For this modified map, still denoted by $f_{o}$, the origin becomes a Lebesgue point of both functions $D f_{o}$ and $\Psi\left[K_{I}\left(x, f_{o}\right)\right]$. What we have gained here is that $f_{o}$ is an isometry near the origin; we have $D f_{o}(0)=\mathbf{I}$ and $\Psi\left[K_{I}\left(0, f_{o}\right)\right]=\Psi(1)$. For every $0<\epsilon<r<1$ we consider the radial mapping $g: \overline{\mathbb{B}} \rightarrow \overline{\mathbb{B}}$ defined by the rule

$$
g(x)=x \rho(|x|)
$$

where

$$
\rho(|x|)= \begin{cases}\epsilon & \text { if }|x| \leqslant r \\ \frac{\epsilon-r}{1-r}+\frac{1-\epsilon}{1-r}|x| & \text { if } r \leqslant|x| \leqslant 1 .\end{cases}
$$

The distortion function of $g$ can be explicitly computed $[\mathbf{1 6}$, p. 114] giving the following estimate:

$$
K_{I}(x, g) \leqslant \begin{cases}1 & \text { for }|x| \leqslant r \\ \frac{2}{1-r} & \text { for } r \leqslant|x| \leqslant 1\end{cases}
$$

Note that $g(x)=\epsilon x$ for $|x| \leqslant r$. Now the following composite map is a legitimate competitor for our variational integral:

$$
f(x)=f_{o}(g(x)) .
$$

The chain rule is valid for quasiconformal mappings, so we can write

$$
D f(x)=D f_{o}(g(x)) \cdot D g(x)=\epsilon D f_{o}(\epsilon x), \quad \text { for }|x| \leqslant r .
$$

Hence we obtain the following estimate of the inner distortion function of $f$ :

$$
K_{I}(x, f) \leqslant \begin{cases}K_{I}\left(\epsilon x, f_{o}\right) & \text { for }|x| \leqslant r \\ \frac{2 K}{1-r} & \text { for } r \leqslant|x| \leqslant 1\end{cases}
$$

where $K=\left\|K_{I}\left(x, f_{o}\right)\right\|_{\infty}$. We may now evaluate the variational integral

$$
\int_{\mathbb{B}} \Psi\left[K_{I}(x, f)\right] d x \leqslant \int_{|x| \leqslant r} \Psi\left[K_{I}\left(\epsilon x, f_{o}\right)\right] d x+\int_{r \leqslant|x|<1} \Psi\left(\frac{2 K}{1-r}\right) d x .
$$

Hence

$$
f_{\mathbb{B}} \Psi\left[K_{I}(x, f)\right] d x \leqslant f_{|y| \leqslant \epsilon} \Psi\left[K_{I}\left(y, f_{o}\right)\right] d y+\left(1-r^{n}\right) \Psi\left(\frac{2 K}{1-r}\right) .
$$

Using the supposed sublinear growth of $\Psi$ we find that the latter term goes to zero as $r \rightarrow 1$. In the first term we let $\epsilon$ go to zero. Since the origin is the Lebesgue point of $\Psi\left[K_{I}\left(x, f_{o}\right)\right]$, the integral mean converges to $\Psi\left[K_{I}\left(0, f_{o}\right)\right]=\Psi(1)$. In conclusion, the infimum at (5.4) does not exceed $\Psi(1)$. On the other hand the integrand is always greater than or equal to $\Psi(1)$. Thus (5.4) holds. 
Finally, any minimizer of finite distortion must satisfy the point-wise equation

$$
\Psi\left[K_{I}(x, f)\right]=\Psi(1) \quad \text { a.e. in } \mathbb{B}
$$

or, equivalently, $K_{I}(x, f)=1$ a.e. in $\mathbb{B}$ because $\Psi$ is strictly increasing. Thus $f$ is conformal by the Liouville Theorem $[\mathbf{1 6}]$. This shows that for arbitrary boundary values, other than those of a conformal mapping of course, there can be no minimizers to the problem (5.1).

\section{The $\mathcal{L}^{1}$-Grötzsch problem, $n=2$}

In this section we present an $\mathcal{L}^{1}$-variant of the celebrated Grötzsch extremal problem for mappings between rectangles, [8]. In this section we confine our discussion to two dimensions. Let $\mathbb{Q}$ be the unit square in the plane, that is,

$$
\mathbb{Q}=[0,1] \times[0,1] \subset \mathbb{R}^{2},
$$

and $\mathbb{Q}^{\prime}$ be a rectangle

$$
\mathbb{Q}^{\prime}=[0,2] \times[0,1] \subset \mathbb{R}^{2}
$$

We shall show that

$$
\min _{f \in \mathcal{F}} \iint_{\mathbb{Q}} \mathbb{K}(z, f)|d z|^{2}=\frac{5}{4}
$$

where $\mathcal{F}$ consists of homeomorphisms $f: \overline{\mathbb{Q}} \rightarrow \overline{\mathbb{Q}^{\prime}}$ in the Sobolev class $\mathcal{W}_{\text {loc }}^{1,1}\left(\mathbb{Q}, \mathbb{R}^{2}\right)$ of integrable distortion taking vertices into vertices. Our goal is to show that this free boundary value problem still has unique extremal. Before jumping into the proof of this result we demonstrate that uniqueness is lost for $K(z, f)$.

Theorem 6.2. The minimization problem

$$
\min _{f \in \mathcal{F}} \iint_{\mathbb{Q}} K(z, f)|d z|^{2}
$$

has infinitely many extremals.

Proof. Suppose $f \in \mathcal{F}$; we first show that

$$
2 \leqslant \iint_{\mathbb{Q}} K(z, f)|d z|^{2} .
$$

To see this we note that

$$
2 \leqslant \int_{0}^{1}|D f(x+i y)| d x \quad \text { for almost all } y \in[0,1],
$$

and then after integrating over $y$ we find that

$$
2 \leqslant \iint_{\mathbb{Q}}|D f(z)||d z|^{2}=\iint_{\mathbb{Q}} \sqrt{K(z, f)} \sqrt{J(z, f)}|d z|^{2} .
$$

Upon squaring, Hölder's inequality implies that

$$
4 \leqslant \iint_{\mathbb{Q}} K(z, f)|d z|^{2} \cdot \iint_{\mathbb{Q}} J(z, f)|d z|^{2} .
$$


Since $f: \mathbb{Q} \rightarrow \mathbb{Q}^{\prime}$ is a homeomorphism of the Sobolev class $\mathcal{W}_{\text {loc }}^{1,1}\left(\mathbb{Q}, \mathbb{R}^{2}\right)$, it follows that

$$
\iint_{\mathbb{Q}} J(x, f) d x \leqslant\left|\mathbb{Q}^{\prime}\right|
$$

where equality holds if $f$ maps sets of zero measure into sets of zero measure. The claim (6.4) follows.

For every $0 \leqslant a<1$, the piece-wise linear map

$$
g(z)= \begin{cases}x+i y & \text { if } z \in[0, a] \times[0,1] \\ \frac{2-a}{1-a} x-\frac{a}{1-a}+i y & \text { if } z \in[a, 1] \times[0,1]\end{cases}
$$

is a minimizer. Indeed, $g$ is the identity if $0 \leqslant x \leqslant a$, while for $x \leqslant a<1$ we have

$$
D g(z)=\left(\begin{array}{cc}
\frac{2-a}{1-a} & 0 \\
0 & 1
\end{array}\right), \quad|D g| \equiv \frac{2-a}{1-a} \text { whence } K(z, g) \equiv \frac{2-a}{1-a} .
$$

The integral of $K(z, g)$ does not depend on $a$; indeed

$$
\iint_{\mathbb{Q}} K(z, g)|d z|^{2}=a \cdot 1+(1-a) \frac{2-a}{1-a}=2 .
$$

This completes the proof of Theorem 6.2.

The situation is dramatically different for the distortion function $\mathbb{K}$.

Theorem 6.12. The minimization problem (6.1) has a unique extremal.

Proof. Let $f: \overline{\mathbb{Q}} \rightarrow \overline{\mathbb{Q}^{\prime}}$ be any admissible mapping, that is, $f \in \mathcal{F}$. Using complex notation

$$
f(z)=u(x, y)+i v(x, y), \quad z=x+i y,
$$

we observe that, for almost every $0<y<1$,

$$
\int_{0}^{1} u_{x}(x+i y) d x=u(1+i y)-u(i y)=2,
$$

while, for almost every $0<x<1$,

$$
\int_{0}^{1} v_{y}(x+i y) d x=u(x+i)-u(x)=1 .
$$

Further integration yields

$$
\iint_{\mathbb{Q}} u_{x} d x d y=2 \text { and } \iint_{\mathbb{Q}} v_{y} d x d y=1 .
$$

We combine these equations in one weighted sum and use the Schwarz inequality to obtain

$$
\begin{aligned}
5 & =\iint_{\mathbb{Q}}\left(2 u_{x}+v_{y}\right) d x d y \leqslant \iint_{\mathbb{Q}} \sqrt{5} \sqrt{u_{x}^{2}+v_{y}^{2}} d x d y \\
& \leqslant \iint_{\mathbb{Q}} \sqrt{5} \sqrt{u_{x}^{2}+v_{y}^{2}+u_{y}^{2}+v_{x}^{2}} d x d y \\
& =\sqrt{10} \iint_{\mathbb{Q}}\left\|D^{\sharp} f\right\|=\sqrt{10} \iint_{\mathbb{Q}} \sqrt{\mathbb{K}(z, f)} \sqrt{J(z, f)}|d z|^{2} .
\end{aligned}
$$


Upon squaring both sides, Hölder's inequality implies

$$
25 \leqslant 10\left|\mathbb{Q}^{\prime}\right| \iint_{\mathbb{Q}} \mathbb{K}(z, f)|d z|^{2}=20 \iint_{\mathbb{Q}} \mathbb{K}(z, f)|d z|^{2} .
$$

In other words,

$$
\iint_{\mathbb{Q}} \mathbb{K}(z, f)|d z|^{2} \geqslant \frac{5}{4} \quad \text { for all } f \in \mathcal{F} .
$$

As expected, equality occurs when $f(z)=2 x+i y$. We need only show that the equation

$$
\iint_{\mathbb{Q}} \mathbb{K}(z, f)|d z|^{2}=\frac{5}{4} \quad \text { for } f \in \mathcal{F} \quad \text { yields } \quad f=2 x+i y .
$$

To this end, we must examine when equalities occur in the chain of the above estimates. First, (6.18) holds as an equality if and only if

$$
u_{y} \equiv v_{x} \equiv 0
$$

meaning that $u$ depends only on $x$, and $v$ depends only on $y$. Recall that (6.17) came from using the Schwartz inequality. This forces the following relation:

$$
2 v_{y}=u_{x} \quad \text { a.e. in } \mathbb{Q} \text {. }
$$

As $u_{x}$ and $v_{y}$ depend on different variables, we infer that $u_{x}$ and $v_{y}$ are constant functions. This leaves only one possibility,

$$
u(x, y)=2 x \quad \text { and } \quad v(x, y)=y
$$

as claimed.

\section{The Grötzsch problem, $n>2$}

In higher dimensions there are several distortion functions to investigate. Given $\ell=1,2, \ldots, n-1$ we shall consider the minimization problem

$$
\min _{f \in \mathcal{F}} \int_{\mathbb{Q}} \mathbb{K}_{\ell}(x, f) d x
$$

where $\mathcal{F}$ consists of homeomorphisms $f=\left(f^{1}, \ldots, f^{n}\right): \overline{\mathbb{Q}} \rightarrow \overline{\mathbb{Q}^{\prime}}$ of the Sobolev class $\mathcal{W}_{\text {loc }}^{1, p}\left(\Omega, \mathbb{R}^{n}\right)$, where $p>\ell$, with finite distortion for which $\mathbb{K}_{\ell}(x, f)$ is integrable. Here $\mathbb{Q}$ and $\mathbb{Q}^{\prime}$ are rectangular boxes,

$$
\mathbb{Q}=\left[0, a_{1}\right] \times \ldots \times\left[0, a_{n}\right] \subset \mathbb{R}^{n}, \quad \mathbb{Q}^{\prime}=\left[0, a_{1}^{\prime}\right] \times \ldots \times\left[0, a_{n}^{\prime}\right] \subset \mathbb{R}^{n} .
$$

We shall assume that $f$ maps every $(n-1)$-dimensional face

$$
\left[0, a_{1}\right] \times \ldots \times\left[0, a_{k-1}\right] \times\{a\} \times\left[0, a_{k+1}\right] \times \ldots \times\left[0, a_{n}\right],
$$

where $a=0$ or $a=a_{k}$, into the corresponding face

$$
\left[0, a_{1}^{\prime}\right] \times \ldots \times\left[0, a_{k-1}^{\prime}\right] \times\left\{a^{\prime}\right\} \times\left[0, a_{k+1}^{\prime}\right] \times \ldots \times\left[0, a_{n}^{\prime}\right],
$$

where $a^{\prime}=0$ or $a^{\prime}=a_{k}^{\prime}$, respectively. This actually implies that $f$ maps every $\ell$-dimensional face, $\ell=0,1, \ldots, n$, of $\mathbb{Q}$ into the corresponding $\ell$-dimensional face of $\mathbb{Q}^{\prime}$. 
One example of a mapping in $\mathcal{F}$ is the linear map

$$
g(x)=\left(\lambda_{1} x_{1}, \ldots, \lambda_{n} x_{n}\right) \quad \text { with } \lambda_{k}=\frac{a_{k}^{\prime}}{a_{k}} .
$$

THEOREM 7.6. For each $\ell=1,2, \ldots, n-1$, the minimization problem (7.1) has exactly one solution, namely the linear map $g$.

Proof. We consider the $\ell$ th exterior power of the differential matrix

$$
D^{\ell \times \ell} f(x) \in \mathbb{R}^{\left(\begin{array}{l}
n \\
l
\end{array}\right) \times\left(\begin{array}{l}
n \\
l
\end{array}\right)}
$$

defined for almost every $x=\left(x_{1}, \ldots, x_{n}\right) \in \mathbb{Q}$. To every multi-index $I=\left(i_{1}, i_{2}, \ldots, i_{\ell}\right)$, with $1 \leqslant i_{1}<\ldots<i_{\ell} \leqslant n$, there corresponds the diagonal entry of $D^{\ell \times \ell} f$, namely

$$
\frac{\partial\left(f^{i_{1}}, \ldots, f^{i_{\ell}}\right)}{\partial\left(x_{i_{1}}, \ldots, x_{i_{\ell}}\right)} .
$$

We shall first prove the following inequality:

$$
\lambda_{i_{1}} \cdot \ldots \cdot \lambda_{i_{\ell}} \leqslant f_{\mathbb{Q}}\left|\frac{\partial\left(f^{i_{1}}, \ldots, f^{i_{\ell}}\right)}{\partial\left(x_{i_{1}}, \ldots, x_{i_{\ell}}\right)}\right| d x .
$$

To simplify the writing consider the case $\left(i_{1}, i_{2}, \ldots, i_{\ell}\right)=(1,2, \ldots, \ell)$. Fix the remaining variables $x_{\ell+1}=c_{\ell+1}, \ldots, x_{n}=c_{n}$ and define the mapping

$$
F\left(x_{1}, x_{2}, \ldots, x_{\ell}\right)=\left(f^{1}\left(x_{1}, \ldots, x_{\ell}, c_{\ell+1}, \ldots, c_{n}\right), \ldots, f^{\ell}\left(x_{1}, \ldots, x_{\ell}, c_{\ell+1}, \ldots, c_{n}\right)\right)
$$

on the closed $\ell$-dimensional rectangle

$$
\overline{\mathbb{U}}:=\left[0, a_{1}\right] \times \ldots \times\left[0, a_{\ell}\right] \subset \mathbb{R}^{\ell} .
$$

This mapping is valued in the closed $\ell$-dimensional rectangle

$$
\overline{\mathbb{U}^{\prime}}:=\left[0, a_{1}^{\prime}\right] \times \ldots \times\left[0, a_{\ell}^{\prime}\right] \subset \mathbb{R}^{\ell} .
$$

By elementary topological arguments we see that $F: \overline{\mathbb{U}} \rightarrow \overline{\mathbb{U}^{\prime}}$ is a continuous surjective map, for every parameter

$$
c=\left(c_{\ell+1}, \ldots, c_{n}\right) \in \mathbb{W}:=\left[0, a_{\ell+1}\right] \times \ldots \times\left[0, a_{n}\right] .
$$

Moreover, for almost every $c \in \mathbb{W}$ this map $F$ belongs to the Sobolev class $\mathcal{W}_{\text {loc }}^{1, p}\left(\mathbb{U}, \mathbb{U}^{\prime}\right)$, where we emphasize that $p>\ell=\operatorname{dim} \mathbb{U}$. Now the $\ell \times \ell$-minor $\partial\left(f^{1}, \ldots, f^{\ell}\right) / \partial\left(x_{1}, \ldots, x_{\ell}\right)$ is nothing other than the Jacobian determinant of $F$. At this point we appeal to geometric measure theory (see for instance $[\mathbf{2}$, Theorem $8.3]$ ), to deduce that

$$
\int_{\mathbb{U}}\left|\frac{\partial\left(f^{1}, \ldots, f^{\ell}\right)}{\partial\left(x_{1}, \ldots, x_{\ell}\right)}\right| d x_{1} \ldots d x_{\ell} \geqslant\left|\mathbb{U}^{\prime}\right| .
$$

Integrating with respect to the parameter $c \in \mathbb{W}$, by Fubini's theorem, we obtain

$$
\int_{\mathbb{U} \times \mathbb{W}}\left|\frac{\partial\left(f^{1}, \ldots, f^{\ell}\right)}{\partial\left(x_{1}, \ldots, x_{\ell}\right)}\right| d x \geqslant\left|\mathbb{U}^{\prime}\right||\mathbb{W}|=a_{1}^{\prime} \ldots a_{\ell}^{\prime} \cdot a_{\ell+1} \ldots a_{n}
$$

which is the same as (7.9). 
We now multiply $(7.9)$ by the product $\lambda_{i_{1}} \ldots \lambda_{i_{\ell}}$ and sum with respect to all multi-indices

$$
\begin{aligned}
& \sum_{1 \leqslant i_{1}<\ldots<i_{\ell} \leqslant n}\left(\lambda_{i_{1}} \ldots \lambda_{i_{\ell}}\right)^{2} \\
& \leqslant \int_{\mathbb{Q}_{1 \leqslant i_{1}<\ldots<i_{\ell} \leqslant n}} \lambda_{i_{1}} \ldots \lambda_{i_{\ell}}\left|\frac{\partial\left(f^{i_{1}}, \ldots, f^{i_{\ell}}\right)}{\partial\left(x_{i_{1}}, \ldots, x_{i_{\ell}}\right)}\right| d x \\
& \leqslant f_{\mathbb{Q}}\left[\sum_{1 \leqslant i_{1}<\ldots<i_{\ell} \leqslant n}\left(\lambda_{i_{1}} \ldots \lambda_{i_{\ell}}\right)^{2}\right]^{1 / 2} \\
& \quad \times\left[\sum_{1 \leqslant i_{1}<\ldots<i_{\ell} \leqslant n}\left|\frac{\partial\left(f^{i_{1}}, \ldots, f^{i_{\ell}}\right)}{\partial\left(x_{i_{1}}, \ldots, x_{i_{\ell}}\right)}\right|^{2}\right]^{1 / 2} d x .
\end{aligned}
$$

Hence,

$$
\begin{aligned}
& {\left[\sum_{1 \leqslant i_{1}<\ldots<i_{\ell} \leqslant n}\left(\lambda_{i_{1}} \ldots \lambda_{i_{\ell}}\right)^{2}\right]^{1 / 2}} \\
& \leqslant f_{\mathbb{Q}}\left[\sum_{1 \leqslant i_{1}<\ldots<i_{\ell} \leqslant n}\left|\frac{\partial\left(f^{i_{1}}, \ldots, f^{i_{\ell}}\right)}{\partial\left(x_{i_{1}}, \ldots, x_{i_{\ell}}\right)}\right|^{2}\right]^{1 / 2} \\
& \leqslant f_{\mathbb{Q}}\left[\sum_{\substack{1 \leqslant i_{1}<\ldots<i_{\ell} \leqslant n \\
1 \leqslant j_{1}<\ldots<j_{\ell} \leqslant n}}\left|\frac{\partial\left(f^{i_{1}}, \ldots, f^{i_{\ell}}\right)}{\partial\left(x_{j_{1}}, \ldots, x_{j_{\ell}}\right)}\right|^{2}\right]^{1 / 2} .
\end{aligned}
$$

The reader may wish to notice that we have added the missing off-diagonal entries of $D^{\ell \times \ell} f$ to give us the Hilbert-Schmidt norm of $D^{\ell \times \ell} f$ :

$$
\begin{aligned}
{\left[\left(\begin{array}{c}
n \\
\ell
\end{array}\right)^{-1} \sum_{1 \leqslant i_{1}<\ldots<i_{\ell} \leqslant n}\left(\lambda_{i_{1}} \ldots \lambda_{i_{\ell}}\right)^{2}\right]^{1 / 2} } & \leqslant f_{\mathbb{Q}}\left\|D^{\ell \times \ell} f(x)\right\| d x \\
& \leqslant\left(f_{\mathbb{Q}} J(x, f) d x\right)^{\ell / n}\left(f_{\mathbb{Q}} \mathbb{K}_{\ell}(x, f) d x\right)^{(n-\ell) / n} .
\end{aligned}
$$

The latter follows by Hölder's inequality and the identity

$$
\left\|D^{\ell \times \ell} f\right\|=J(x, f)^{\ell / n} \mathbb{K}_{\ell}(x, f)^{1-\ell / n} .
$$

In conclusion,

$$
\begin{aligned}
f_{\mathbb{Q}} \mathbb{K}_{\ell}(x, f) d x & \geqslant\left(\lambda_{1} \ldots \lambda_{n}\right)^{\ell /(\ell-n)} \cdot\left[\left(\begin{array}{l}
n \\
\ell
\end{array}\right)^{-1} \sum_{1 \leqslant i_{1}<\ldots<i_{\ell} \leqslant n}\left(\lambda_{i_{1}} \ldots \lambda_{i_{\ell}}\right)^{2}\right]^{n /(2 n-2 \ell)} \\
& =f_{\mathbb{Q}} \mathbb{K}_{\ell}(x, g) d x .
\end{aligned}
$$

Thus the linear map $g$ is a minimizer. As in the previous section we shall establish the uniqueness of the minimizer by examining all the steps in the above computation. First, (7.18) holds as an equality if and only if the matrix $D^{\ell \times \ell} f$ is diagonal. We now need an algebraic lemma.

Lemma 7.1. Let $A \in \mathbb{R}^{n \times n}$ be a non-singular matrix whose $\ell$ th exterior power $A^{\ell \times \ell} \in \mathbb{R}^{\left(\begin{array}{l}n \\ \ell\end{array}\right) \times\left(\begin{array}{l}n \\ \ell\end{array}\right)}$ is diagonal. Then $A$ is diagonal. 
Proof. Set $A=\left[A_{j}^{i}\right]$ and note that $\operatorname{det} A^{\ell \times \ell}=(\operatorname{det} A)^{\ell} \neq 0$. Our goal is to show that $A_{\beta}^{\alpha}=0$ whenever $\alpha \neq \beta$. We choose a multi-index $I=\left\{i_{1}, \ldots, i_{\ell}\right\}$, with $1 \leqslant i_{1}<\ldots<i_{\ell} \leqslant n$ containing $\alpha$ but not $\beta$. We shall actually prove that the whole vector

$$
\mathfrak{b}=\left(A_{i_{1}}^{\beta}, A_{i_{2}}^{\beta}, \ldots, A_{i_{\ell}}^{\beta}\right) \in \mathbb{R}^{\ell},
$$

in which $A_{\alpha}^{\beta}$ is one of the coordinates, is equal to zero. To this end consider the $\ell \times \ell$-submatrix of $A$ :

$$
M=\left(\begin{array}{cccc}
A_{i_{1}}^{i_{1}} & A_{i_{2}}^{i_{1}} & \cdots & A_{i_{\ell}}^{i_{1}} \\
\vdots & \vdots & \ddots & \vdots \\
A_{i_{1}}^{i_{\ell}} & A_{i_{2}}^{i_{\ell}} & \cdots & A_{i_{\ell}}^{i_{\ell}}
\end{array}\right)
$$

This is a non-singular matrix because $\operatorname{det} M$ is an entry of a non-singular diagonal matrix $A^{\ell \times \ell}$. We may solve the equation $M \mathfrak{a}=\mathfrak{b}$ for $\mathfrak{a}=\left(a_{1}, a_{2}, \ldots, a_{\ell}\right) \in \mathbb{R}^{\ell}$. By Cramer's formula the coordinates of $\mathfrak{a}$ are given by

$$
a_{k}=\frac{\operatorname{det} M_{k}}{\operatorname{det} M}, \quad \text { for } k=1,2, \ldots, \ell
$$

where $M_{k}$ is a matrix obtained from $M$ by replacing its $k$ th column by the vector $\mathfrak{b}=\left(A_{i_{1}}^{\beta}, A_{i_{2}}^{\beta}, \ldots, A_{i_{\ell}}^{\beta}\right)$. Since $\beta$ does not belong to the set $I$, $\operatorname{det} M_{k}$ is an outdiagonal entry of $A^{\ell \times \ell}$ and, therefore, is equal to zero. We conclude that each $a_{k}=0$; hence $\mathfrak{b}=M^{-1} \mathfrak{a}=0$, completing the proof of Lemma 7.1.

Using this lemma we infer that $D f$ is also diagonal, the case $\operatorname{det} D f=0$ being trivial. Obviously, the differential matrix can be diagonal only when each of the coordinate functions depends on its own variable, $f^{1}=f^{1}\left(x_{1}\right), \ldots, f^{n}=f^{n}\left(x_{n}\right)$. Furthermore, (7.16) becomes an equality only when the minors

$$
\left|\frac{\partial\left(f^{i_{1}}, \ldots, f^{i_{\ell}}\right)}{\partial\left(x_{i_{1}}, \ldots, x_{i_{\ell}}\right)}\right|=\left|\frac{\partial f^{i_{1}}}{\partial x_{i_{1}}}\right| \ldots\left|\frac{\partial f^{i_{\ell}}}{\partial x_{i_{\ell}}}\right|
$$

remain, at almost every point, in the same proportion as the products $\lambda_{i_{1}} \ldots \lambda_{i_{\ell}}$. This is because the Schwarz inequality has been used in (7.16). In other words, there is a measurable function $\lambda=\lambda(x)$ such that

$$
\left|\frac{\partial f^{i_{1}}}{\partial x_{i_{1}}}\right| \ldots\left|\frac{\partial f^{i_{\ell}}}{\partial x_{i_{\ell}}}\right|=\lambda_{i_{1}} \ldots \lambda_{i_{\ell}} \lambda(x) .
$$

The left-hand side depends only on the variables $x_{i_{1}}, \ldots, x_{i_{\ell}}$, forcing $\lambda$ to be a constant. On the other hand, each coordinate function $f^{i}=f^{i}\left(x_{i}\right)$, defined for $0 \leqslant x_{i} \leqslant a_{i}$, must be monotone because $f\left(x_{1}, x_{2}, \ldots, x_{n}\right)=\left(f^{1}\left(x_{1}\right), \ldots, f^{n}\left(x_{n}\right)\right)$ is a homeomorphism. We then see that the derivative of each coordinate function is constant. This leaves the only possibility that

$$
f(x)=\left(\lambda_{1} x_{1}, \ldots, \lambda_{n} x_{n}\right),
$$

as desired. 


\section{Linear boundary values}

We then turn to Problem 1 from the introduction, that is, minimizing of the integral (2.6) under fixed boundary values $f_{0}$. We show first that in the case when the boundary data is linear and the domain has a boundary of finite $(n-1)$ dimensional Hausdorff measure, the problem has a unique minimizer in the full class $\mathcal{W}_{\text {loc }}^{1,1}\left(\Omega, \mathbb{R}^{n}\right)$. This rectifiability assumption allows us to use a general form of Stokes' Theorem in the setting of Sobolev functions.

TheOREM 8.1. Let $\Omega \subset \mathbb{R}^{n}$ be a bounded domain with $(n-1)$-rectifiable boundary and let $\Psi:[0, \infty) \rightarrow[0, \infty)$ be convex with $\Psi^{\prime}(1) \geqslant 1$.

Given any homeomorphism $f: \bar{\Omega} \rightarrow \overline{\Omega^{\prime}}$ of finite distortion, which coincides on $\partial \Omega$ with an orientation-preserving affine map $f_{o}: \mathbb{R}^{n} \rightarrow \mathbb{R}^{n}$, we have

$$
\int_{\Omega} \mathbb{K}_{\Psi}(x, f) d x \geqslant \int_{\Omega} \mathbb{K}_{\Psi}\left(x, f_{o}\right) d x .
$$

Equality occurs if and only if $f=f_{o}$ on $\Omega$.

Proof. We begin with the subgradient inequality

$$
\Psi(t)-\Psi\left(t_{0}\right) \geqslant \Psi^{\prime}\left(t_{0}\right)\left(t-t_{0}\right),
$$

valid for every $t, t_{0} \in[1, \infty)$. Here $\Psi^{\prime}\left(t_{0}\right) \geqslant \Psi^{\prime}(1)>0$ is any subgradient of $\Psi$ at $t_{0}$.

We use the subgradient inequality at the point

$$
t_{0}=\mathbb{K}\left(D f_{o}\right)=\frac{\left\|D f_{o}\right\|^{n}}{\operatorname{det} D f_{o}} \geqslant 1,
$$

where the inequality holds since $f_{o}$ is non-singular with $\operatorname{det} D f_{o}>0$.

We claim that for almost every $x \in \Omega$ we have

$$
\begin{aligned}
& \mathbb{K}_{\Psi}(D f(x))-\mathbb{K}_{\Psi}\left(D f_{o}\right) \\
& \quad \geqslant\left\langle\Gamma, D f(x)-D f_{o}\right\rangle+\gamma\left[\operatorname{det} D f_{o}-\operatorname{det} D f(x)\right],
\end{aligned}
$$

where

$$
\Gamma=\Gamma\left(D f_{o}\right)=\Psi^{\prime}\left(t_{0}\right) \frac{\left\|D f_{o}\right\|^{n-2}}{\operatorname{det} D f_{o}} D f_{o} \in \mathbb{R}_{+}^{n \times n}
$$

and

$$
\gamma=\gamma\left(D f_{o}\right)=\Psi^{\prime}\left(t_{0}\right) \frac{\left\|D f_{o}\right\|^{n}}{\left(\operatorname{det} D f_{o}\right)^{2}} \in \mathbb{R}_{+}^{n} .
$$

In fact, as $f \in \mathcal{W}_{\text {loc }}^{1,1}\left(\Omega, \mathbb{R}^{n}\right)$, it has partial derivatives at almost every $x \in \Omega$, and at such points $x$ we have two possibilities. Since $f$ is a mapping of finite distortion, either $\|D f(x)\|=0$ or $\operatorname{det} D f(x)>0$.

In the first case, by definition, $\mathbb{K}_{\Psi}(D f(x))=1$ so that

$$
\begin{aligned}
\mathbb{K}_{\Psi}(D f(x))-\mathbb{K}_{\Psi}\left(D f_{o}\right) & =\Psi(1)-\Psi\left(\mathbb{K}\left(D f_{o}\right)\right) \\
& \geqslant \Psi^{\prime}\left(t_{0}\right)\left(1-\mathbb{K}\left(D f_{o}\right)\right)>-\Psi^{\prime}\left(t_{0}\right) \mathbb{K}\left(D f_{o}\right)
\end{aligned}
$$

since $\Psi^{\prime}\left(t_{0}\right)>0$. As

$$
\left\langle\Gamma, D f_{o}\right\rangle=n \Psi^{\prime}\left(t_{0}\right) \mathbb{K}\left(D f_{o}\right)
$$


and

$$
\gamma \operatorname{det} D f_{o}=\Psi^{\prime}\left(t_{0}\right) \mathbb{K}\left(D f_{o}\right)
$$

the lower bound (8.5) follows whenever $\|D f(x)\|=0$ or $\operatorname{det} D f(x)=0$.

In the second case, if $x \in \Omega$ is such a point that $\operatorname{det} D f(x)>0$, we have from the subgradient inequality:

$$
\mathbb{K}_{\Psi}(D f(x))-\mathbb{K}_{\Psi}\left(D f_{o}\right) \geqslant \Psi^{\prime}\left(t_{0}\right)\left(\mathbb{K}(D f(x))-\mathbb{K}\left(D f_{o}\right)\right) .
$$

We then need the inequality (A.11) from the appendix which implies that

$$
\begin{aligned}
\mathbb{K}(D f(x))-\mathbb{K}\left(D f_{o}\right)= & \frac{\|D f(x)\|^{n}}{\operatorname{det} D f(x)}-\frac{\left\|D f_{o}\right\|^{n}}{\operatorname{det} D f_{o}} \\
\geqslant & n \frac{\left\|D f_{o}\right\|^{n-1}}{\operatorname{det} D f_{o}}\left(\|D f(x)\|-\left\|D f_{o}\right\|\right) \\
& -\frac{\left\|D f_{o}\right\|^{n}}{\left(\operatorname{det} D f_{o}\right)^{2}}\left(\operatorname{det} D f(x)-\operatorname{det} D f_{o}\right) .
\end{aligned}
$$

Furthermore, we estimate by using the Schwarz inequality

$$
\|D f(x)\|-\left\|D f_{o}\right\| \geqslant \frac{1}{n}\left\langle\frac{D f_{o}}{\left\|D f_{o}\right\|}, D f(x)-D f_{o}\right\rangle,
$$

where we recall that $\langle A, B\rangle=\operatorname{Tr}\left\langle A^{t} B\right\rangle$ and $\|A\|^{2}=n^{-1} \operatorname{Tr}\left\langle A^{t} A\right\rangle$. Equality here holds at $x$ if and only if

$$
D f(x)=\lambda(x) D f_{o}, \quad \text { where the scalar function } \lambda(x) \geqslant 0 .
$$

Now combining the estimates (8.9), (8.10) and (8.11) yields the desired bound and shows that (8.5) holds at almost every point $x \in \Omega$.

For the next step we wish to integrate the terms of (8.5) over the domain $\Omega$. However, since $f$ is only assumed to be in $\mathcal{W}_{\text {loc }}^{1,1}\left(\Omega, \mathbb{R}^{n}\right)$ we restrict ourselves to an increasing sequence of compact subdomains $\Omega_{j} \Subset \Omega$, for $j=1,2, \ldots$, such that $\bigcup \Omega_{j}=\Omega$ and $\sup _{j}\left|\partial \Omega_{j}\right|<\infty$. This is possible since $\partial \Omega$ is $(n-1)$-rectifiable.

Concerning the first term in the right-hand side of (8.5) we observe, using Stokes' formula, that

$$
\left|\int_{\Omega_{j}}\left[D f(x)-D f_{o}\right] d x\right| \leqslant C \int_{\partial \Omega_{j}}\left|f-f_{o}\right| \rightarrow 0
$$

as $j \rightarrow \infty$. Furthermore, in (8.5) the matrix function $D f(x)-D f_{o}$ appears in an inner product with a constant matrix. We then infer that upon integration, this first term converges to zero as $j \rightarrow \infty$.

For the second term in the right-hand side of (8.5) we argue by using the inequality

$$
\int_{\Omega_{j}} \operatorname{det} D f(x) d x \leqslant\left|f\left(\Omega_{j}\right)\right| \leqslant|f(\Omega)|=\left|f_{o}(\Omega)\right|=\int_{\Omega} \operatorname{det} D f_{o} d x .
$$

Note that it is at this point that we have exploited the assumption that $f$ is a homeomorphism in $\mathcal{W}_{\text {loc }}^{1,1}\left(\Omega, \mathbb{R}^{n}\right)$. In conclusion, the limit of the integral of this 
second term is non-negative. This shows that

$$
\int_{\Omega} \mathbb{K}_{\Psi}(x, f) d x \geqslant \int_{\Omega} \mathbb{K}_{\Psi}\left(x, f_{o}\right) d x .
$$

In order to have equality in (8.15) we must have equality in (8.8) and in (8.11), at almost all points $x \in \Omega$. This forces first $\operatorname{det} D f(x)>0$ and then (8.12) to hold almost everywhere. Equation (8.12), on the other hand, reads also as

$$
D g(x)=\lambda(x) I, \quad \text { where } g=f_{o}^{-1} \circ f .
$$

This implies that $\lambda=\lambda_{o}$ is a constant and that $g(x)=\lambda_{o} I$. Thus $f(x)=$ $\lambda_{o} f_{o}(x)+$ const. Finally, since $f=f_{o}$ on $\partial \Omega$, we conclude that $f=f_{o}$ on $\Omega$, as claimed.

\section{An identity}

Variations of the identity we are about to formulate are fairly well known in geometric function theory; see $[\mathbf{2}, \mathbf{2 0}]$. However, what we need is not explicitly stated there and for the convenience of the reader, and because it is quite important in what follows, we present a complete proof of it. Throughout this section, $\Omega$ and $\Omega^{\prime}$ will be bounded domains in $\mathbb{R}^{n}$, with $n \geqslant 2$.

TheOREM 9.1. Let $f \in \mathcal{W}_{\text {loc }}^{1, n}\left(\Omega, \Omega^{\prime}\right)$ be a homeomorphism of finite distortion with

$$
\int_{\Omega} K_{I}(x, f) d x<\infty
$$

Then the inverse map $h: \Omega^{\prime} \rightarrow \Omega$ belongs to $\mathcal{W}^{1, n}\left(\Omega^{\prime}, \Omega\right)$ and

$$
\int_{\Omega^{\prime}}|D h(y)|^{n} d y=\int_{\Omega} K_{I}(x, f) d x .
$$

Proof. Fix a test mapping $\varphi \in \mathcal{C}_{0}^{\infty}\left(\Omega^{\prime}, \mathbb{R}^{n}\right)$. For $k=1,2, \ldots, n$ we consider the Sobolev mappings $F_{k} \in \mathcal{W}_{\text {loc }}^{1, n}\left(\Omega, \mathbb{R}^{n}\right)$,

$$
F_{k}(x)=\left(f^{1}, \ldots, f^{k-1}, \omega, f^{k+1}, \ldots, f^{n}\right), \quad \omega(x)=\sum_{i=1}^{n} x_{i} \varphi^{i}(f(x)) .
$$

As the $k$ th coordinate function has compact support, we see that

$$
\int_{\Omega} J\left(x, F_{k}\right) d x=0 .
$$

A lengthy, though standard, computation shows that the vector field

$$
V(x)=\left(J\left(x, F_{1}\right), \ldots, J\left(x, F_{n}\right)\right)
$$

can be written as:

$$
\left[D^{\sharp} f(x)\right] \varphi(f(x))+J(x, f)\left[\left(D^{t} \varphi\right)(f(x))\right] x .
$$


Since $\int_{\Omega} V(x) d x=0 \in \mathbb{R}^{n}$, we obtain

$$
\int_{\Omega}\left[\left(D^{t} \varphi\right)(f(x))\right] x J(x, f) d x=-\int_{\Omega}\left[D^{\sharp} f(x)\right] \varphi(f(x)) d x .
$$

At this point we appeal to an old result of Reshetnyak [30, Corollary 1, p. 182] concerning change of variables via a homeomorphism of the Sobolev class $\mathcal{W}_{\text {loc }}^{1, n}\left(\Omega, \mathbb{R}^{n}\right)$. We make the substitution $y=f(x)$ in the integral on the left-hand side to obtain

$$
\int_{\Omega^{\prime}}\left[\left(D^{t} \varphi\right)(y)\right] h(y) d y=-\int_{\Omega}\left[D^{\sharp} f(x)\right] \varphi(f(x)) d x .
$$

So far we have only exploited the fact that $f \in \mathcal{W}_{\text {loc }}^{1, n}\left(\Omega, \mathbb{R}^{n}\right)$ is a homeomorphism. Before using the identity (9.9) let us recall that $f$, being a mapping of finite distortion, satisfies the following condition:

$$
D^{\sharp} f(x)=0 \quad \text { if and only if } \quad J(x, f)=0 .
$$

Hence

$$
\begin{aligned}
\left|\int_{\Omega^{\prime}}\left[\left(D^{t} \varphi\right)(y)\right] h(y) d y\right| & \leqslant \int_{\Omega}\left|D^{\sharp} f(x)\right||\varphi(f(x))| d x \\
& =\int_{\Omega} \sqrt[n]{K_{I}(x, f)} J(x, f)^{(n-1) / n}|\varphi(f(x))| d x \\
& \leqslant\left(\int_{\Omega} K_{I}(x, f) d x\right)^{1 / n}\left(\int_{\Omega}|\varphi(f)|^{n /(n-1)} J(\cdot, f)\right)^{(n-1) / n} \\
& =\left\|K_{I}(x, f)\right\|_{\mathcal{L}^{1}(\Omega)}^{1 / n}\|\varphi\|_{\mathcal{L}^{n /(n-1)}\left(\Omega^{\prime}\right)},
\end{aligned}
$$

where we have made the substitution $y=f(x)$ again. This estimate tells us exactly that $h \in \mathcal{W}^{1, n}\left(\Omega^{\prime}, \Omega\right)$ and its differential satisfies

$$
\int_{\Omega^{\prime}}|D h(y)|^{n} d y \leqslant \int_{\Omega} K_{I}(x, f) d x .
$$

Now, knowing that $h \in \mathcal{W}^{1, n}\left(\Omega^{\prime}, \Omega\right)$, we can legitimately use change of variables to obtain the identity

$$
\int_{\Omega^{\prime}}|D h(y)|^{n} d y=\int_{\Omega}|D h(f(x))|^{n} J(x, f) d x=\int_{\Omega} K_{I}(x, f) d x,
$$

completing the proof of Theorem 9.1.

REMARK 9.1. We still owe the reader an explanation why

$$
|D h(f(x))|^{n} J(x, f)=K_{I}(x, f)
$$

almost everywhere. To see this we first observe that both $f$ and $h$ are differentiable almost everywhere. This elegant result belongs to Väisälä [32]. The chain rule $D h(f(x)) D f(x)=\mathbf{I}$ shows that $J(x, f)>0$ and $J(y, h)>0$ almost everywhere since both $f$ and $h$ preserve sets of zero measure. (A theorem of Reshetnyak [30, Corollary 1, p.182], tells us that a homeomorphism $f$ of Sobolev class $W_{\text {loc }}^{1, n}$ preserves sets of measure zero. Precisely, if $|E|=0$, then $|f(E)|=0$. 
Since in our case the inverse map $h$ also belongs to $W_{\text {loc }}^{1, n}, h$ also preserves sets of measure zero. The measure of the image is equal to the integral of the Jacobian determinant, again by this same result.) Now, the formula is a direct consequence of the definition of the inner distortion,

$$
K_{I}(x, f):=\frac{\left|D^{\sharp} f(x)\right|^{n}}{J(x, f)^{n-1}}=\left|D^{-1} f(x)\right|^{n} J(x, f)=|D h(f(x))|^{n} J(x, f) .
$$

\section{The $\mathcal{L}^{1}$-theory with Dirichlet data}

The minimisation problem with general boundary values is of course considerably deeper than the problem for linear data.

We first study homeomorphisms of finite distortion $f: \bar{\Omega} \rightarrow \overline{\Omega^{\prime}}$ between bounded domains $\Omega, \Omega^{\prime} \subset \mathbb{R}^{n}$, such that the inner distortion function of $f$ is integrable.

In view of Theorem 9.1 , for $f \in \mathcal{W}_{\text {loc }}^{1, n}\left(\Omega, \Omega^{\prime}\right)$ the minimization of

$$
\int_{\Omega} K_{I}(x, f) d x
$$

is closely related to a well-known variational problem for the inverse mapping $h=f^{-1}: \Omega \rightarrow \Omega^{\prime}$.

The Dirichlet Problem. Given a mapping $h_{o} \in \mathcal{W}^{1, n}\left(\Omega^{\prime}, \mathbb{R}^{n}\right)$ minimize the energy

$$
\int_{\Omega^{\prime}}|D h(y)|^{n} d y<\infty
$$

over the class of all mappings $h \in h_{o}+\mathcal{W}_{0}^{1, n}\left(\Omega^{\prime}, \mathbb{R}^{n}\right)$.

While the existence of the minimizer is guaranteed by the principles of convex analysis, the uniqueness is a delicate issue due to the lack of strict convexity of the operator norm. One way out of this is to replace the operator norm by the mean Hilbert-Schmidt norm. Therefore, we consider the following variational integral:

$$
\mathcal{E}[h]=\int_{\Omega^{\prime}}\|D h(y)\|^{n} d y .
$$

The advantage of using this functional is that the minimization problem

$$
\min \int_{\Omega^{\prime}}\|D h(y)\|^{n} d y, \quad h \in h_{o}+\mathcal{W}_{0}^{1, n}\left(\Omega^{\prime}, \mathbb{R}^{n}\right)
$$

admits a unique solution for all Dirichlet data $h_{o} \in \mathcal{W}^{1, n}\left(\Omega^{\prime}, \mathbb{R}^{n}\right)$. Moreover, the minimizer is the unique solution to the $n$-harmonic equation

$$
\operatorname{Div}\|D h\|^{n-2} D h=0, \quad \text { for } h \in h_{o}+\mathcal{W}_{0}^{1, n}\left(\Omega^{\prime}, \mathbb{R}^{n}\right) .
$$

This equation simply means that

$$
\int_{\Omega^{\prime}}\|D h\|^{n-2}\langle D h \mid D \varphi\rangle=0
$$

for every test mapping $\varphi \in \mathcal{C}_{0}^{\infty}\left(\Omega^{\prime}, \mathbb{R}^{n}\right)$. 
To formulate an analogue of Theorem 9.1 we make use of the mean Hilbert-Schmidt variant of the inner distortion

$$
\mathbb{K}_{I}(x, f)=\frac{\left\|D^{\sharp} f(x)\right\|^{n}}{J(x, f)^{n-1}}=\mathbb{K}_{n-1}(D f(x)) ;
$$

cf. $\S 3$.

THEOREM 10.8. Let $f \in \mathcal{W}_{\text {loc }}^{1, n}\left(\Omega, \Omega^{\prime}\right)$ be a homeomorphism of finite distortion with

$$
\int_{\Omega} \mathbb{K}_{I}(x, f) d x<\infty .
$$

Then the inverse map $h: \Omega^{\prime} \rightarrow \Omega$ belongs to $\mathcal{W}^{1, n}\left(\Omega^{\prime}, \Omega\right)$ and

$$
\int_{\Omega^{\prime}}\|D h(y)\|^{n} d y=\int_{\Omega} \mathbb{K}_{I}(x, f) d x .
$$

Proof. The chain of inequalities at (9.11), together with Theorem 9.1, guarantees that $h$ lies in the Sobolev class $\mathcal{W}^{1, n}\left(\Omega^{\prime}, \Omega\right)$. The only thing we have to worry about here is the identity (10.10). This follows by replacing (9.14) with the following computation:

$$
\mathbb{K}_{I}(x, f)=\left\|D^{-1} f(x)\right\|^{n} J(x, f)=\|D h(f(x))\|^{n} J(x, f),
$$

which proves the identity (10.10).

To apply Theorem 10.8 to our variational problem we need to recall some of the considerable literature on the existence and topological properties of harmonic maps between planar domains. We refer the reader to the recent book of Duren [6]. In particular, according to the fundamental theorem of Radó [29], Kneser [18] and Choquet $[\mathbf{3}]$, if $\Omega \subset \mathbb{R}^{2}$ is a bounded convex domain, then each homeomorphism $h_{o}: \partial \Omega^{\prime} \rightarrow \partial \Omega$ has a unique continuous extension $h: \overline{\Omega^{\prime}} \rightarrow \bar{\Omega}$ which is univalent and maps $\Omega^{\prime}$ harmonically to $\Omega$. Then, by a theorem of Lewy $[\mathbf{2 3}]$, the univalent harmonic map has a non-vanishing Jacobian. Its inverse is therefore a real analytic diffeomorphism.

We now consider the class $\mathcal{F}=\mathcal{F}\left(\Omega, \Omega^{\prime}\right)$ of $\mathcal{W}_{\text {loc }}^{1,2}\left(\Omega, \mathbb{R}^{2}\right)$-regular homeomorphisms $f: \bar{\Omega} \rightarrow \overline{\Omega^{\prime}}$ of finite distortion for which $\mathbb{K}(z, f)$ is integrable in $\Omega$.

ThEOREM 10.12. Let $\Omega \subset \mathbb{R}^{2}$ be a convex domain and $f_{o} \in \mathcal{F}\left(\Omega, \Omega^{\prime}\right)$. Then the minimization problem

$$
\min _{f \in \mathcal{F}} \iint_{\Omega} \mathbb{K}(z, f)|d z|^{2}, \quad f=f_{0} \text { on } \partial \Omega,
$$

has a unique solution. This extremal map is a $\mathcal{C}^{\infty}$-diffeomorphism whose inverse is harmonic in $\Omega^{\prime}$.

Proof. Let $\mathcal{H}=\mathcal{H}\left(\Omega^{\prime}, \Omega\right)$ denote the class of inverse mappings $h=f^{-1}: \overline{\Omega^{\prime}} \rightarrow \bar{\Omega}$ where $f \in \mathcal{F}\left(\Omega, \Omega^{\prime}\right)$. Thus, in particular, $h_{0}=f_{0}^{-1} \in \mathcal{H}\left(\Omega^{\prime}, \Omega\right)$. In light of Theorem 
11.1 we are reduced, equivalently, to the Dirichlet problem

$$
\min _{f \in \mathcal{H}} \iint_{\Omega}\|D h\|^{2}, \quad h=h_{0} \text { on } \partial \Omega
$$

The existence and uniqueness of the minimizer in the Sobolev class $h_{o}+\mathcal{W}_{0}^{1,2}\left(\Omega^{\prime}, \mathbb{R}^{2}\right)$ is well known. The only point to make is that such a minimizer lies in $\mathcal{H}\left(\Omega^{\prime}, \Omega\right)$ by the Radó-Kneser-Choquet and Lewy theorems.

Recently Hencl, Koskela and Onninen [12] showed, by proving the main identity (9.3) in this class when $n=2$, that the minimisation problem of Theorem 10.12 has a unique minimiser in the class $\mathcal{W}_{\text {loc }}^{1,1}\left(\Omega, \Omega^{\prime}\right)$.

Corollary 10.1. The extremal map $f: \Omega \rightarrow \Omega^{\prime}$ solves the quasilinear Beltrami equation

$$
\frac{\partial f}{\partial \bar{z}}=\mu(f(x)) \frac{\partial f}{\partial z}
$$

where $\mu: \Omega^{\prime} \rightarrow \mathbb{B}$ is an anti-analytic function valued in the unit disk.

Proof. As $f$ is a diffeomorphism with positive Jacobian,

$$
J(z, f)=\left|f_{z}\right|^{2}-\left|f_{\bar{z}}\right|^{2}>0
$$

and thus $\left|f_{\bar{z}} / f_{z}\right|<1$. Let us define $\mu: \Omega^{\prime} \rightarrow \mathbb{B}$ by the equation (10.15). We need only show that $\mu$ is anti-analytic. For this reason we consider the inverse map $h(\xi)=f^{-1}(\xi)$ to write

$$
\mu(\xi)=-\frac{h_{\bar{\xi}}}{\overline{h_{\xi}}}
$$

Hence

$$
\frac{\partial \mu}{\partial \xi}=-\left(\frac{h_{\bar{\xi}}}{\overline{h_{\xi}}}\right)=\frac{-h_{\xi \bar{\xi}} \overline{h_{\xi}}+h_{\bar{\xi}} \overline{h_{\xi \bar{\xi}}}}{\left(\overline{h_{\xi}}\right)^{2}}=0,
$$

as claimed.

Now the following is immediate.

Corollary 10.2 (maximum principle). Let $\mathbb{K}(z, f)$ denote the inner distortion of the extremal map. Then

$$
\max _{\mathbb{U}} \mathbb{K}(x, f) \leqslant \max _{\partial \mathbb{U}} \mathbb{K}(x, f)
$$

for every $\mathbb{U} \subset \Omega$.

\section{The traces of mappings with integrable distortion}

Theorem 10.12 demands, for the sake of completeness, that we give necessary and sufficient conditions for a homeomorphism $f_{0}: \partial \Omega \rightarrow \partial \Omega^{\prime}$ to admit an extension $f: \bar{\Omega} \rightarrow \overline{\Omega^{\prime}}$ which lies in $\mathcal{F}\left(\Omega, \Omega^{\prime}\right)$, the class of $\mathcal{W}_{\text {loc }}^{1,2}\left(\Omega, \mathbb{R}^{2}\right)$-regular homeomorphisms $f: \bar{\Omega} \rightarrow \overline{\Omega^{\prime}}$ of finite distortion for which $\mathbb{K}(z, f)$ is integrable in 
$\Omega$. Recall that for the $\mathcal{L}^{\infty}$ minimisation problem the requisite notion is that of quasisymmetry, though in this case there is a surprise; see Theorem 11.27.

TheOREM 11.1. Suppose $\Gamma=\partial \Omega$ and $\Gamma^{\prime}=\partial \Omega^{\prime}$ are $\mathcal{C}^{1}$-regular Jordan curves. $A$ necessary and sufficient condition that a homeomorphism $f_{o}: \Gamma \rightarrow \Gamma^{\prime}$ should extend to an $f: \bar{\Omega} \rightarrow \overline{\Omega^{\prime}}$, where $f \in \mathcal{F}\left(\Omega, \Omega^{\prime}\right)$, is that the double integral

$$
L_{\Gamma}\left(f_{o}\right):=-\frac{1}{\pi} \iint_{\Gamma \times \Gamma} \log \left|f_{o}(z)-f_{o}(w)\right| d z d \bar{w}
$$

converges absolutely. That is,

$$
\iint_{\Gamma \times \Gamma}|\log | f_{o}(z)-f_{o}(w)|||d z||d w|<\infty .
$$

Among all such extensions of $f_{o}$ there is one which maps $\Omega$ diffeomorphically onto $\Omega^{\prime}$.

Using the Riemann mapping theorem we reduce to the case when both $\Omega$ and $\Omega^{\prime}$ are disks in $\mathbb{C}$. Because of the $\mathcal{C}^{1}$-regularity of $\Gamma=\partial \Omega$ and $\Gamma^{\prime}=\partial \Omega^{\prime}$ neither the hypotheses nor the assertion of this theorem will be affected by such a change of variables. Therefore, we shall have established Theorem 11.1 once we prove the following more precise special case of it.

TheOREM 11.4. Theorem 11.1 holds when $\Omega^{\prime}$ is a disk and $\Omega$ has the additional property of being convex. In this case, for every extension of $f_{0}$ to an $f \in \mathcal{F}\left(\Omega, \Omega^{\prime}\right)$, we have

$$
-\frac{1}{\pi} \iint_{\Gamma \times \Gamma} \log \left|f_{o}(z)-f_{o}(w)\right| d z d \bar{w} \leqslant \iint_{\Omega} \mathbb{K}(z, f)|d z|^{2} .
$$

Equality occurs only when $f=h^{-1}$, where $h: \overline{\Omega^{\prime}} \rightarrow \bar{\Omega}$ is the unique harmonic extension of $f_{0}^{-1}: \Gamma^{\prime} \rightarrow \Gamma$. This extremal extension turns out to be a diffeomorphism.

REMARK. Our results are reminiscent of ideas of Douglas [5], characterizing boundary functions whose harmonic extension have finite Dirichlet energy; see Verchota [33] for more. The Douglas condition for $h_{o}=f_{o}^{-1}$ reads as

$$
\iint_{\Gamma^{\prime} \times \Gamma^{\prime}}\left|\frac{h_{o}(\xi)-h_{o}(\zeta)}{\xi-\zeta}\right|^{2}|d \xi||d \zeta|<\infty
$$

Proof. Observe that (11.5) is invariant under translation and rescaling of $\Omega^{\prime}$, so we may assume that $\Omega^{\prime}$ is the unit disk, $\Omega^{\prime}=\mathbb{D} \subset \mathbb{C}$. Consider the inverse homeomorphism $h_{o}: \partial \mathbb{D} \rightarrow \partial \Omega$. As shown in the previous section, $f_{o}$ admits an extension to $f \in \mathcal{F}\left(\Omega, \Omega^{\prime}\right)$ if and only if the Poisson extension $h: \overline{\mathbb{D}} \rightarrow \bar{\Omega}$ has finite energy. Moreover, in this case the inverse map $f=h^{-1}$ provides us with one of the desired extensions of $f_{o}$ to $f \in \mathcal{F}\left(\Omega, \Omega^{\prime}\right)$. 
We begin with integral representation formulas of the complex derivatives of the harmonic map $h$ in terms of $f_{o}: \Gamma \rightarrow \partial \mathbb{D}$ :

$$
\begin{aligned}
& \frac{\partial h}{\partial a}=\frac{1}{2 \pi i} \int_{\Gamma} \frac{d z}{f_{o}(z)-a}, \\
& \frac{\partial h}{\partial \bar{a}}=\frac{1}{2 \pi i} \int_{\Gamma} \frac{d z}{\bar{a}-\overline{f_{o}(z)}},
\end{aligned}
$$

for every $a \in \mathbb{D}$. We give the proof only for (11.7); the second identity follows in much the same way. Consider an exhaustion of $\Omega$ by smooth domains $\Omega_{1} \Subset \Omega_{2} \Subset \ldots \subset \Omega$, such that

$$
a \in f\left(\Omega_{1}\right) \Subset f\left(\Omega_{2}\right) \Subset \ldots \subset \mathbb{D} .
$$

We have

$$
\begin{aligned}
\int_{\Gamma} \frac{d z}{f_{o}(z)-a} & =\lim _{n \rightarrow \infty} \int_{\partial \Omega_{n}} \frac{d z}{f(z)-a}=\lim _{n \rightarrow \infty} \int_{C_{n}} \frac{d h(\xi)}{\xi-a} \\
& =\lim _{n \rightarrow \infty} \int_{C_{n}} \frac{h_{\xi} d \xi}{\xi-a}+\lim _{n \rightarrow \infty} \int_{C_{n}} \frac{h_{\bar{\xi}} d \bar{\xi}}{\xi-a},
\end{aligned}
$$

where the curves $C_{n}=f\left(\partial \Omega_{n}\right)$ approach $\partial \mathbb{D}$, uniformly as $n \rightarrow \infty$. We do not claim here that the lengths of $C_{n}$ stay bounded. Since $h_{\xi}$ is an analytic function in $\mathbb{D}$, the first integral is independent of the curve $C_{n}$ and equals $2 \pi i \partial h / \partial a$, by Cauchy's formula. Concerning the second integral, it would be equal to zero if $C_{n}$ was a circle. Indeed, we would have

$$
\int_{|\xi|=\rho} \frac{h_{\bar{\xi}} d \bar{\xi}}{\xi-a}=\int_{|\xi|=\rho} \frac{\bar{\xi} h_{\bar{\xi}} d \bar{\xi}}{\rho^{2}-a \bar{\xi}}=0,
$$

by Cauchy's theorem for anti-analytic functions. The above arguments suggest imbedding every $f\left(\Omega_{n}\right)$ in a disk, say $f\left(\Omega_{n}\right) \subset \mathbb{D}_{n} \Subset \mathbb{D}$. We can now express the curve integral by the area integral by using Stokes' formula

$$
\begin{aligned}
\int_{C_{n}} \frac{h_{\bar{\xi}} d \bar{\xi}}{\xi-a} & =\int_{C_{n}} \frac{h_{\bar{\xi}} d \bar{\xi}}{\xi-a}-\int_{\partial \mathbb{D}_{n}} \frac{h_{\bar{\xi}} d \bar{\xi}}{\xi-a}=\iint_{\mathbb{D}_{n} \backslash f\left(\Omega_{n}\right)} d\left[\frac{h_{\bar{\xi}} d \bar{\xi}}{\xi-a}\right] \\
& =-\iint_{\mathbb{D}_{n} \backslash f\left(\Omega_{n}\right)} \frac{h_{\bar{\xi}} d \xi \wedge d \bar{\xi}}{(\xi-a)^{2}} .
\end{aligned}
$$

Hölder's inequality yields

$$
\left|\int_{C_{n}} \frac{h_{\bar{\xi}} d \bar{\xi}}{\xi-a}\right| \leqslant C\left(\iint_{\mathbb{D} \backslash f\left(\Omega_{n}\right)}|D h|^{2}\right)^{1 / 2} \rightarrow 0 \quad \text { as } n \rightarrow \infty
$$

completing the proof of (11.7). Having disposed of formulas (11.7) and (11.8) we can, since $|D h| \in \mathcal{L}^{2}(\mathbb{D})$, compute the Dirichlet integral of $h$ over an arbitrary disk $\mathbb{D}_{r}=\{\xi:|\xi|<r\}$, with $0<r<1$,

$$
\iint_{\mathbb{D}_{r}}|D h|^{2}=2 \iint_{|a| \leqslant r}\left|\frac{\partial h}{\partial a}\right|^{2}+2 \iint_{|a| \leqslant r}\left|\frac{\partial h}{\partial \bar{a}}\right|^{2} .
$$


The computation of the first integral goes as follows:

$$
\left|\frac{\partial h}{\partial a}\right|^{2}=\frac{1}{4 \pi^{2}} \iint_{\Gamma \times \Gamma} \frac{d z d \bar{w}}{\left[f_{o}(z)-a\right]\left[\overline{f_{o}(w)}-\bar{a}\right]} .
$$

Hence, by Fubini's theorem,

$$
\iint_{|a| \leqslant r}\left|\frac{\partial h}{\partial a}\right|^{2}=\frac{1}{4 \pi^{2}} \iint_{\Gamma \times \Gamma}\left(\iint_{|a| \leqslant r} \frac{|d a|^{2}}{\left[f_{o}(z)-a\right]\left[\overline{f_{o}(w)}-\bar{a}\right]}\right) d z d \bar{w} .
$$

A tedious (but elementary) computation, developing the integrand as a power series, yields an explicit expression for the area integral

$$
\iint_{|a| \leqslant r} \frac{|d a|^{2}}{(\xi-a)(\bar{\zeta}-\bar{a})}=-\pi \log \left(1-r^{2} \bar{\xi} \zeta\right)
$$

where $|\xi|=|\zeta|=1$. We substitute this value into the latter formula to obtain

$$
2 \iint_{|a| \leqslant r}\left|\frac{\partial h}{\partial a}\right|^{2}=-\frac{1}{2 \pi} \iint_{\Gamma \times \Gamma} \log \left(1-r^{2} f_{o}(z) \overline{f_{o}(w)}\right) d z d \bar{w} .
$$

Similar arguments to those above show that

$$
2 \iint_{|a| \leqslant r}\left|\frac{\partial h}{\partial \bar{a}}\right|^{2}=-\frac{1}{2 \pi} \iint_{\Gamma \times \Gamma} \log \left(1-r^{2} \overline{f_{o}(z)} f_{o}(w)\right) d z d \bar{w} .
$$

These two equations add up to

$$
\iint_{\mathbb{D}_{r}}|D h|^{2}=-\frac{1}{2 \pi} \iint_{\Gamma \times \Gamma} \log \left|1-r^{2} f_{o}(z) \overline{f_{o}(w)}\right|^{2} d z d \bar{w}
$$

which, in view of the identity (10.10), can be stated as

$$
\begin{gathered}
-\frac{1}{\pi} \iint_{\Gamma \times \Gamma} \log \left|r^{2}-f_{o}(z) \overline{f_{o}(w)}\right| d z d \bar{w} \\
=\iint_{\mathbb{D}_{r}}\|D h\|^{2} \\
=2 \iint_{f^{-1}\left(\mathbb{D}_{r}\right)} \mathbb{K}(z, f)|d z|^{2} .
\end{gathered}
$$

It is now clear that the integral in the left-hand side increases with $r$. Letting $r$ go to 1 we see that the limit exists if and only if $\mathbb{K}(z, f)$ is integrable. The only point remaining concerns the equivalence of the following two properties of the boundary map $f_{o}: \Gamma \rightarrow \partial \mathbb{D}$ : the existence of this limit and the absolute convergence of the integral

$$
\iint_{\Gamma \times \Gamma}|\log | f_{o}(z)-f_{o}(w)|||d z||d w| .
$$

It is clear that, regardless of the regularity of $\Gamma$, the absolute convergence of the integral at (11.20) implies that

$$
\lim _{r \nearrow 1} \iint_{\Gamma \times \Gamma} \log \left|r^{2}-f_{o}(z) \overline{f_{o}(w)}\right| d z d \bar{w}=\iint_{\Gamma \times \Gamma} \log \left|f_{o}(z)-f_{o}(w)\right| d z d \bar{w}
$$

by the Lebesgue Dominated Convergence Theorem. For the converse, we need 
$\mathcal{C}^{1}$-regularity of $\Gamma$. Suppose that the above limit exists. Since the integrand is invariant under the interchange of the variables $z$ and $w$, we may replace the complex area element $d z d \bar{w}$ by the real one $\operatorname{Re}(d z d \bar{w})$. However, on $\mathcal{C}^{1}$-regular curves this latter element is comparable with $|d z||d w|$ when $z$ is sufficiently close to $w$, say

$$
\frac{1}{2}|d z||d w| \leqslant \operatorname{Re}(d z d \bar{w}) \leqslant 2|d z||d w|,
$$

provided $|z-w| \leqslant \epsilon$. The interested reader may wish to observe that this estimate fails for the cube $\Omega=[0,1] \times[0,1]$. Indeed, near the corner $(0,0)$ we may take $z=x$ and $w=i y$ to obtain

$$
\operatorname{Re}(d z d \bar{w})=-\operatorname{Re}(i d x d y)=0 .
$$

On the other hand (11.22), with 2 replaced by some positive number, remains valid, for example, for polygons with obtuse angles. Now, for $\mathcal{C}^{1}$-regular curves, in view of (11.22), the existence of the limit and equality at (11.21) becomes equivalent to the absolute convergence of the integral at (11.2), as desired.

EXAMPLE 11.1. Consider a homeomorphism of the unit circle onto itself $f_{o}$ : $\partial \mathbb{D} \rightarrow \partial \mathbb{D}$ given by

where

$$
f_{o}\left(e^{i \theta}\right)=e^{i \Phi(\theta)} \quad \text { for }-\pi<\theta \leqslant \pi
$$

$$
\Phi(\theta)=\pi \operatorname{sgn} \theta e^{1-\pi^{2} / \theta^{2}}
$$

The reader may wish to verify that the double integral

$$
\int_{0}^{\pi} \int_{-\pi}^{0} \log \left|e^{i \Phi(\alpha)}-e^{i \Phi(\beta)}\right| d \alpha d \beta
$$

diverges. As a corollary, we see that $f_{o}$ has no homeomorphic extension into the unit disk with integrable distortion.

Note that in the proof of Theorem 11.4 we did not really have to use $\mathcal{C}^{1}$-regularity of the convex domain $\Omega$; we could have used the limit formula at (11.21) instead of the integral at (11.5). As $\Omega$ is convex, its boundary $\Gamma=\partial \Omega$ is Lipschitz and, therefore, a rectifiable Jordan curve. We leave the details of such an extension of Theorem 11.4 to arbitrary convex domains to the reader.

Finally in this section, we wish to observe that given quasiconformal boundary data, even for the disk $\mathbb{D}$, the minimiser of the $\mathcal{L}^{1}$-problem is seldom quasiconformal.

Theorem 11.27. Let $f_{o}: \mathbb{D} \rightarrow \mathbb{D}$ be quasiconformal and $\mathcal{F}_{o}$ as in Theorem 10.12. Then the unique minimiser of the problem

$$
\min _{f \in \mathcal{F}} \iint_{\Omega} \mathbb{K}(z, f)|d z|^{2}, \quad f=f_{0} \text { on } \partial \mathbb{D}
$$

is quasiconformal if and only if $f_{o}$ is bi-Lipschitz.

Proof. Set $g_{o}=f_{o}^{-1}$. We know that the minimiser $f$ exists and its inverse $h$ is the unique harmonic extension of $g_{o} \mid \partial \mathbb{D}$. If $f$ is quasiconformal, then so too is $h$. 
However a theorem of Pavlovic [28] states that the Poisson extension of the boundary values $g_{o}$ of a quasiconformal mapping is quasiconformal if and only if the map $g_{o}$ is bi-Lipschitz. Thus $f_{o}$ is bi-Lipschitz.

Actually [28] points out that the quasiconformality of the Poisson extension is equivalent to the boundary values themselves being bi-Lipschitz or that the Hilbert Transform of their derivative lies in $\mathcal{L}^{\infty}$.

\section{Exponentially integrable distortion}

In this section we shall be concerned with the variational integrals (2.6) when $\Psi(t)=e^{\lambda t-\lambda}$ for some fixed parameter $\lambda>0$. If $\Omega$ and $\Omega^{\prime}$ are bounded domains, we shall consider the family $\mathcal{F}$ of all homeomorphisms $f: \bar{\Omega} \rightarrow \overline{\Omega^{\prime}}$ of a finite distortion such that

$$
\int_{\Omega} e^{\lambda \mathbb{K}(x, f)} d x<\infty
$$

We fix $f_{o} \in \mathcal{F}$ and denote by $\mathcal{F}_{o}$ the class of all $f \in \mathcal{F}$ which coincide with $f_{o}$ on $\partial \Omega$. For convenience we assume, moreover, that $f_{o}$ extends to a neighborhood of $\bar{\Omega}$ as a homeomorphism of finite distortion satisfying (12.1).

TheOREM 12.2. The minimization problem

$$
\min _{f \in \mathcal{F}_{o}} \int_{\Omega} e^{\lambda \mathbb{K}(x, f)} d x=m
$$

has a solution.

As an interesting first point to make we show that the inverse mapping of any $f \in \mathcal{F}_{o}$ has better Sobolev regularity than $f$ itself, namely $f^{-1} \in \mathcal{W}^{1, n}\left(\Omega^{\prime}, \Omega\right)$. This is an improvement of Theorem 10.8, since we do not assume that $f \in \mathcal{W}_{\text {loc }}^{1, n}\left(\Omega, \Omega^{\prime}\right)$.

THEOREM 12.4. Let $f: \Omega \rightarrow \Omega^{\prime}$ be a homeomorphism of finite distortion with

$$
\int_{\Omega} e^{\lambda \mathbb{K}(x, f)} d x<\infty
$$

Then $f$ lies in the Orlicz-Sobolev space $\mathcal{W}^{1, P}\left(\Omega, \Omega^{\prime}\right)$, with $P(t)=t^{n} / \log (e+t)$, and

$$
\int_{\Omega} \frac{\|D f\|^{n}}{\log \left(e+\|D f\|^{n}\right)} \leqslant \frac{1}{\lambda}\left|\Omega^{\prime}\right|+\int_{\Omega} e^{\lambda \mathbb{K}(x, f)} d x .
$$

Moreover, the inverse map $h: \Omega^{\prime} \rightarrow \Omega$ belongs to $\mathcal{W}^{1, n}\left(\Omega^{\prime}, \Omega\right)$ and

$$
\int_{\Omega^{\prime}}\|D h(y)\|^{n} d y=\int_{\Omega} \mathbb{K}_{I}(x, f) d x .
$$

Proof. We follow the arguments of Theorems 10.8 and 9.1. In the proof of Theorem 9.1 we defined auxiliary mappings

$$
F_{k}(x)=\left(f^{1}, \ldots, f^{k-1}, \omega, f^{k+1}, \ldots, f^{n}\right), \quad \omega(x)=\sum_{i=1}^{n} x_{i} \varphi^{i}(f(x)) .
$$


Recall that the assumption $f \in \mathcal{W}_{\text {loc }}^{1, n}\left(\Omega, \mathbb{R}^{n}\right)$ was used to ensure that $F_{k} \in \mathcal{W}_{\text {loc }}^{1, n}\left(\Omega, \mathbb{R}^{n}\right)$. This regularity of $F_{k}$ was important in order to have the identity

$$
\int_{\Omega} J\left(x, F_{k}\right) d x=0 .
$$

This time the assumptions of Theorem 12.4 only guarantee that $f \in \mathcal{W}^{1, P}\left(\Omega, \Omega^{\prime}\right)$. Indeed, for homeomorphisms of the Sobolev class $\mathcal{W}_{\text {loc }}^{1,1}\left(\Omega, \Omega^{\prime}\right)$ we have

$$
\int_{\Omega} J(x, f) d x \leqslant|f(\Omega)|=\left|\Omega^{\prime}\right| .
$$

The distortion inequality

$$
\|D f\|^{n} \leqslant \mathbb{K}(x, f) J(x, f)
$$

yields

$$
\frac{\|D f\|^{n}}{\log \left(e+\|D f\|^{n}\right)} \leqslant \frac{1}{\lambda} J(x, f)+e^{\lambda \mathbb{K}(x, f)}
$$

where we have employed the elementary inequality

$$
a b \leqslant a \log (a+1)+e^{b}-1 \text { for } a, b \geqslant 0 .
$$

Hence

$$
\int_{\Omega} \frac{\|D f\|^{n}}{\log \left(e+\|D f\|^{n}\right)} \leqslant \frac{1}{\lambda}\left|\Omega^{\prime}\right|+\int_{\Omega} e^{\lambda \mathbb{K}(x, f)} d x<\infty .
$$

Thus $f \in \mathcal{W}_{\text {loc }}^{1, P}\left(\Omega, \Omega^{\prime}\right) \subset \mathcal{W}_{\text {loc }}^{1, n-1}\left(\Omega, \Omega^{\prime}\right)$. Hence also $F_{k} \in \mathcal{W}_{\text {loc }}^{1, P}\left(\Omega, \mathbb{R}^{n}\right)$.

Although the Jacobian determinant of $F_{k}$ changes sign, the Jacobian is still locally integrable. Indeed,

where

$$
J\left(x, F_{k}\right) d x=d f^{1} \wedge \ldots \wedge d f^{k-1} \wedge d \omega \wedge d f^{k+1} \wedge \ldots \wedge d f^{n},
$$

$$
d \omega=\sum_{i=1}^{n} \varphi^{i}(f(x)) d x_{i}+\sum_{i=1}^{n} x_{i} \sum_{j=1}^{n} \frac{\partial \varphi^{i}}{\partial y_{j}} d f^{j} .
$$

Hence,

$$
\begin{aligned}
J\left(x, F_{k}\right) d x= & d f^{1} \wedge \ldots \wedge d f^{k-1} \wedge \sum_{i=1}^{n} \varphi^{i}(f(x)) d x_{i} \wedge d f^{k+1} \wedge \ldots \wedge d f^{n} \\
& +\left[\sum_{i=1}^{n} x_{i} \frac{\partial \varphi^{i}(f(x))}{\partial y_{k}}\right] d f^{1} \wedge \ldots \wedge d f^{n}
\end{aligned}
$$

which is in $\mathcal{L}^{1}(\Omega)$ because $f \in \mathcal{W}_{\text {loc }}^{1, n-1}\left(\Omega, \Omega^{\prime}\right)$ and $J(x, f) \in \mathcal{L}^{1}(\Omega)$. Now Lemma 7.8.1 in $[\mathbf{1 6}]$ comes to the rescue as the identity (12.9) still holds.

For (12.7) we appeal to the computation in the proof of Theorem 9.1. The only point is to justify change of variables, for which we need condition (N). This condition has been established in [17] for mappings of exponentially integrable distortion, completing the proof of Theorem 12.4 .

Proof of Theorem 12.2. Let $\left\{f_{j}\right\}$ be a minimizing sequence. That is,

(i) $f_{j}: \bar{\Omega} \rightarrow \overline{\Omega^{\prime}}$ are homeomorphisms which coincide with $f_{o}: \bar{\Omega} \rightarrow \overline{\Omega^{\prime}}$ on $\partial \Omega$,

(ii) $f_{j} \in \mathcal{W}_{\text {loc }}^{1,1}\left(\Omega, \Omega^{\prime}\right)$, 
(iii) $\left\|D f_{j}(x)\right\|^{n} \leqslant \mathbb{K}\left(x, f_{j}\right) J\left(x, f_{j}\right)$ a.e.,

(iv) $\lim _{j \rightarrow \infty} \int_{\Omega} e^{\lambda \mathbb{K}\left(x, f_{j}\right)} d x=m$.

As in (12.13) this yields uniform estimates of the differentials in the Orlicz space $\mathcal{L}^{P}(\Omega)$,

$$
\int_{\Omega} \frac{\left\|D f_{j}\right\|^{n}}{\log \left(e+\left\|D f_{j}\right\|^{n}\right)} \leqslant \frac{1}{\lambda}\left|\Omega^{\prime}\right|+\int_{\Omega} e^{\lambda \mathbb{K}\left(x, f_{j}\right)} d x \leqslant C \quad \text { for } j=1,2, \ldots .
$$

From these estimates we deduce that the $\left\{f_{j}\right\}$ are equicontinuous on $\bar{\Omega}$. The simplest way to see this is, perhaps, as follows. We extend each $f_{j}$ beyond $\bar{\Omega}$ by setting

$$
\tilde{f}_{j}= \begin{cases}f_{j} & \text { on } \bar{\Omega} \\ f_{o} & \text { beyond } \bar{\Omega} .\end{cases}
$$

The extension is possible since $f_{j}=f_{o}$ on $\partial \Omega$ and $f_{o}$ is defined in a neighborhood of $\bar{\Omega}$, as a homeomorphism of finite distortion satisfying (12.1). We can now use the local estimates of $\left[\mathbf{1 4}\right.$, Theorem 1.4] to conclude, with equicontinuity of $\left\{f_{j}\right\}$ on $\bar{\Omega}$.

Ascolli's theorem gives us a subsequence, again denoted by $f_{j}$, such that

$$
f_{j} \rightrightarrows f \quad \text { uniformly on } \bar{\Omega} \text {. }
$$

Hence

$$
D f_{j} \rightarrow D f \quad \text { weakly in } \mathcal{L}^{P}\left(\Omega, \mathbb{R}^{n \times n}\right) .
$$

Consider then the inverse mappings $h_{j}: \overline{\Omega^{\prime}} \rightarrow \bar{\Omega}$. Theorem 12.4 yields

$$
\int_{\Omega^{\prime}}\left\|D h_{j}\right\|^{n}=\int_{\Omega} \mathbb{K}_{I}\left(x, f_{j}\right) d x \leqslant C, \quad \text { for } j=1,2, \ldots
$$

As above, the homeomorphisms $\left\{h_{j}\right\}$ which coincide with the given $h_{o}=f_{o}^{-1}$ on $\partial \Omega^{\prime}$ share a uniform modulus of continuity on $\overline{\Omega^{\prime}}$. Hence, we find that

$$
h_{j} \rightrightarrows h \quad \text { uniformly on } \overline{\Omega^{\prime}} .
$$

This, together with (12.19), implies that $f$ is a homeomorphism, with $h$ as its inverse. It remains to show that

$$
\int_{\Omega} e^{\lambda \mathbb{K}(x, f)} d x=m
$$

To this end, we observe that the integrand is polyconvex; see Appendix A.2. Precisely, we have the following pointwise inequality:

$$
\begin{aligned}
e^{\lambda \mathbb{K}(X)} \geqslant & e^{\lambda \mathbb{K}(A)}+\lambda e^{\lambda \mathbb{K}(A)}\left[\mathbb{K}_{o}(X)-\mathbb{K}_{o}(A)\right] \\
\geqslant & e^{\lambda \mathbb{K}(A)}+\lambda e^{\lambda \mathbb{K}(A)} \cdot \frac{n\|A\|^{n-1}}{\operatorname{det} A}\left\langle\frac{A}{\|A\|}, X-A\right\rangle \\
& -\lambda e^{\lambda \mathbb{K}(A)} \frac{\|A\|^{n}}{(\operatorname{det} A)^{2}}(\operatorname{det} X-\operatorname{det} A)
\end{aligned}
$$

for matrices $A, X \in \mathbb{R}_{+}^{n \times n}$. Given any $\epsilon>0$ we consider the set $\Omega_{\epsilon} \Subset \Omega$ on which

$$
J(x, f) \geqslant \epsilon \text { and }\|D f(x)\| \leqslant 1 / \epsilon .
$$


Moreover,

$$
\bigcup_{\epsilon>0} \Omega_{\epsilon}=\{x \in \Omega: J(x, f)>0\}
$$

For $x \in \Omega_{\epsilon}$, we can write (see (A.14))

$$
\begin{aligned}
e^{\lambda \mathbb{K}\left(x, f_{j}\right)} \geqslant & e^{\lambda \mathbb{K}(x, f)} \\
& +n \lambda e^{\lambda \mathbb{K}(x, f)} \frac{\|D f(x)\|^{n-1}}{J(x, f)}\left\langle\frac{D f(x)}{\|D f(x)\|}, D f_{j}(x)-D f(x)\right\rangle \\
& -\lambda e^{\lambda \mathbb{K}(x, f)} \frac{\|D f(x)\|^{n}}{J(x, f)^{2}}\left[J\left(x, f_{j}\right)-J(x, f)\right]
\end{aligned}
$$

For notational simplicity we introduce measurable bounded coefficients $\mathcal{A} \in \mathcal{L}^{\infty}\left(\Omega_{\epsilon}, \mathbb{R}^{n \times n}\right)$ and $\alpha \in \mathcal{L}^{\infty}\left(\Omega_{\epsilon}\right)$ which enter the right-hand side. Integrating over $\Omega_{\epsilon}$ yields

$$
\begin{aligned}
\int_{\Omega_{\epsilon}} e^{\lambda \mathbb{K}\left(x, f_{j}\right)} d x \geqslant & \int_{\Omega_{\epsilon}} e^{\lambda \mathbb{K}(x, f)} d x+\int_{\Omega_{\epsilon}}\left\langle\mathcal{A}(x), D f_{j}(x)-D f(x)\right\rangle d x \\
& -\int_{\Omega_{\epsilon}} \alpha(x)\left[J\left(x, f_{j}\right)-J(x, f)\right] d x .
\end{aligned}
$$

The last two integrals converge to zero as $j \rightarrow \infty$. By the definition of outer distortion,

$$
\mathbb{K}_{o}(x, f)=1 \leqslant \mathbb{K}_{o}\left(x, f_{j}\right) \quad \text { whenever } J(x, f)=0 .
$$

With this convention in mind we can write

$$
\begin{aligned}
\int_{\Omega_{\epsilon}} e^{\lambda \mathbb{K}(x, f)} d x+\int_{J(x, f)=0} e^{\lambda \mathbb{K}(x, f)} d x \\
\leqslant \liminf _{j \rightarrow \infty}\left[\int_{\Omega_{\epsilon}} e^{\lambda \mathbb{K}\left(x, f_{j}\right)} d x+\int_{J(x, f)=0} e^{\lambda \mathbb{K}\left(x, f_{j}\right)} d x\right] \\
\leqslant \liminf _{j \rightarrow \infty} \int_{\Omega} e^{\lambda \mathbb{K}\left(x, f_{j}\right)} d x=m
\end{aligned}
$$

Letting $\epsilon \rightarrow 0$, we conclude that

$$
\int_{\Omega} e^{\lambda \mathbb{K}(x, f)} d x \leqslant m
$$

REMARK 12.1. It is shown in [19] that in fact $J(x, f)>0$ almost everywhere, so the addition of the integral over the set where $J(x, f)=0$ is redundant.

\section{Variational equations}

Suggested by many problems in the Calculus of Variation we strongly believe that if $\Psi \in \mathcal{C}^{\infty}[1, \infty)$ then the extremals are continuously differentiable, as in the case $\Psi(t)=t$. 
Conjecture 13.1. Suppose $\Psi$ is $\mathcal{C}^{\infty}$-smooth. Then every homeomorphism of finite distortion on a domain $\Omega$ that minimizes the variational integral

$$
\int_{\Omega} \mathbb{K}_{\Psi}(x, f) d x
$$

subject to given boundary values is a $\mathcal{C}^{1, \alpha}$-diffeomorphism in $\Omega$.

Unfortunately, we do not even know whether the minimizers enjoy partial regularity as in the Quasiconvex Calculus of Variations [7].

Under the conjecture we have the following computation. We begin with a variation of the complex Beltrami coefficient

$$
\mu(z, f):=\frac{f_{\bar{z}}}{f_{z}}
$$

where we assume that $f: \bar{\Omega} \rightarrow \overline{\Omega^{\prime}}$ is an orientation-preserving diffeomorphism. Let $\eta \in \mathcal{C}_{0}^{\infty}(\Omega)$ be a complex-valued test function. For all sufficiently small complex parameters $\lambda$ we still have $J(z, f+\lambda \eta)>0$, and $f+\lambda \eta$ enjoys the same boundary values as $f$. The complex differential of $\mu(z, f)$, denoted by $\dot{\mu}=\dot{\mu}(z, f)$, is a $\mathbb{C}$-linear operator on $\mathcal{C}_{0}^{\infty}(\Omega)$. It acts on a test function $\eta \in \mathcal{C}_{0}^{\infty}(\Omega)$ by the rule

$$
\dot{\mu}[\eta]=\dot{\mu}(z, f)[\eta]=\left.\frac{\partial \mu(z, f+\lambda \eta)}{\partial \lambda}\right|_{\lambda=0}=\frac{f_{z} \eta_{\bar{z}}-f_{\bar{z}} \eta_{z}}{\left(f_{z}\right)^{2}} .
$$

Now consider the function

$$
\kappa=\kappa(z, f)=|\mu(z, f)|^{2}=\frac{\left|f_{\bar{z}}\right|^{2}}{\left|f_{z}\right|^{2}} .
$$

Its complex differential is computed by using the chain rule

$$
\dot{\kappa}=\dot{\kappa}(z, f)=\bar{\mu} \dot{\mu} .
$$

More explicitly, for each $\eta \in \mathcal{C}_{0}^{\infty}(\Omega)$,

$$
\dot{\kappa}[\eta]=\dot{\kappa}(z, f)[\eta]=\kappa\left(\frac{\eta_{\bar{z}}}{f_{\bar{z}}}-\frac{\eta_{z}}{f_{z}}\right) .
$$

Next recall the linear distortion function

$$
\mathbb{K}(z, f)=\frac{\left|f_{z}\right|^{2}+\left|f_{\bar{z}}\right|^{2}}{\left|f_{z}\right|^{2}-\left|f_{\bar{z}}\right|^{2}}=\frac{\|D f(z)\|^{2}}{J(z, f)}=\frac{1+\kappa(z, f)}{1-\kappa(z, f)} .
$$

Again by the chain rule we find that

$$
\dot{\mathbb{K}}=\dot{\mathbb{K}}(z, f)=\frac{2 \dot{\kappa}(z, f)}{(1-\kappa)^{2}},
$$

that is,

$$
\dot{\mathbb{K}}[\eta]=\dot{\mathbb{K}}(z, f)[\eta]=\frac{2 \kappa}{(1-\kappa)^{2}}\left(\frac{\eta_{\bar{z}}}{f_{\bar{z}}}-\frac{\eta_{z}}{f_{z}}\right) .
$$

More generally, for every convex $\Psi:[1, \infty) \rightarrow[1, \infty)$ we have the corresponding 
distortion function

$$
\mathbb{K}_{\Psi}=\mathbb{K}_{\Psi}(z, f)=\Psi(\mathbb{K}(z, f))=\Psi\left(\frac{\left|f_{z}\right|^{2}+\left|f_{\bar{z}}\right|^{2}}{\left|f_{z}\right|^{2}-\left|f_{\bar{z}}\right|^{2}}\right)
$$

whose complex differential equals

$$
\begin{aligned}
\dot{\mathbb{K}}_{\Psi}[\eta]=\dot{\mathbb{K}}_{\Psi}(z, f)[\eta] & =\Psi^{\prime}\left(\frac{1+\kappa}{1-\kappa}\right) \frac{2 \kappa}{(1-\kappa)^{2}}\left(\frac{\eta_{\bar{z}}}{f_{\bar{z}}}-\frac{\eta_{z}}{f_{z}}\right) \\
& =\frac{2}{(1-\kappa)^{2}} \Psi^{\prime}\left(\frac{1+\kappa}{1-\kappa}\right)\left(\frac{\overline{f_{\bar{z}}} \eta_{\bar{z}}-\kappa \overline{f_{z}} \eta_{z}}{\left|f_{z}\right|^{2}}\right) .
\end{aligned}
$$

\subsection{The Lagrange-Euler equations}

We want now to discuss the minimizers $f$ of the general variational integrals

$$
\iint_{\Omega} \mathbb{K}_{\Psi}(z, f)|d z|^{2}
$$

If $f$ is also a $\mathcal{C}^{1}(\Omega)$-diffeomorphism, then

$$
\frac{\partial}{\partial \lambda} \int_{\Omega} \mathbb{K}_{\Psi}(z, f+\lambda \eta)|d z|^{2}=0 \quad \text { at } \lambda=0
$$

for every test function $\eta \in \mathcal{C}_{0}^{\infty}(\Omega)$. This gives

$$
\left[\frac{1}{(1-\kappa)^{2}} \Psi^{\prime}\left(\frac{1+\kappa}{1-\kappa}\right) \frac{\overline{f_{\bar{z}}}}{\left|f_{z}\right|^{2}}\right]_{\bar{z}}=\left[\frac{\kappa}{f_{z}(1-\kappa)^{2}} \Psi^{\prime}\left(\frac{1+\kappa}{1-\kappa}\right)\right]_{z}
$$

or equivalently

$$
\frac{\partial}{\partial \bar{z}}\left(\frac{A(\kappa)}{f_{\bar{z}}}\right)=\frac{\partial}{\partial z}\left(\frac{A(\kappa)}{f_{z}}\right)
$$

where

$$
A(\kappa)=\Psi^{\prime}\left(\frac{1+\kappa}{1-\kappa}\right) \frac{2 \kappa}{(1-\kappa)^{2}}=\Psi^{\prime}\left(\frac{\left|f_{z}\right|^{2}+\left|f_{\bar{z}}\right|^{2}}{\left|f_{z}\right|^{2}-\left|f_{\bar{z}}\right|^{2}}\right) \frac{2\left|f_{z}\right|^{2}\left|f_{\bar{z}}\right|^{2}}{\left(\left|f_{z}\right|^{2}-\left|f_{\bar{z}}\right|^{2}\right)^{2}} .
$$

Let us now introduce the so-called conjugate stationary solution $g=g(z)$ in order to express (13.13) as a first order system. From now on we need to assume that $\Omega$ is a simply connected domain.

Note that $A(\kappa) / f_{\bar{z}}$ and $A(\kappa) / f_{z}$ are continuous in $\Omega$. Therefore there is $g \in \mathcal{C}^{1}(\Omega)$, unique up to a constant, such that

$$
\frac{\partial g}{\partial z}=\frac{A(\kappa)}{f_{\bar{z}}}, \quad \frac{\partial g}{\partial \bar{z}}=\frac{A(\kappa)}{f_{z}} .
$$

Notice that $g$ need not be a homeomorphism even supposing that $f$ were. However we do have the following.

LEMma 13.1. The minimizer $f$ and its conjugate stationary function $g$ have the same complex Beltrami coefficient,

$$
g_{\bar{z}}=\mu(z) g_{z} \quad \text { with } g_{z} f_{\bar{z}}=g_{\bar{z}} f_{z}=A(\kappa)>0
$$

for almost every $z \in \Omega$. 
To every extremal mapping $f: \Omega \rightarrow \Omega^{\prime}$ there corresponds a holomorphic function $F: \Omega^{\prime} \rightarrow \mathbb{C}$ defined by

$$
F(\xi)=g\left(f^{-1}(\xi)\right)
$$

where $g$ denotes the conjugate function to $f$. Indeed, since $f$ is a homeomorphism, we can always express $g(z)=F(f(z))$, for some mapping $F: \Omega^{\prime} \rightarrow \mathbb{C}$. Now, $F$ is holomorphic because $f$ and $g$ have the same Beltrami coefficient. The chain rule gives the following relations:

$$
g_{z}=F^{\prime}(f(z)) f_{z}, \quad g_{\bar{z}}=F^{\prime}(f(z)) f_{\bar{z}} .
$$

Hence

$$
F^{\prime}=\frac{g_{z}}{f_{z}}=\frac{g_{\bar{z}}}{f_{\bar{z}}}=\frac{A(\kappa)}{f_{\bar{z}} f_{z}} .
$$

We recall the derivatives of the inverse map $h(\xi)=f^{-1}(\xi)$,

$$
h_{\bar{\xi}}(\xi)=-f_{\bar{z}}(z) J(\xi, h), \quad h_{\xi}(\xi)=\overline{f_{z}(z)} J(\xi, h),
$$

and compute

$$
k \frac{\left|F^{\prime}(\xi)\right|}{F^{\prime}(\xi)}=k \frac{f_{\bar{z}} f_{z}}{\left|f_{\bar{z}}\right|\left|f_{z}\right|}=\frac{f_{\bar{z}}}{\overline{f_{z}}}=-\frac{h_{\bar{\xi}}}{h_{\xi}}
$$

where

$$
k=k(z, f)=|\mu(z, f)|=|\mu(\xi, h)|=k(\xi, h) .
$$

Corollary 13.1. Let $f: \Omega \rightarrow \Omega^{\prime}$ be a $\mathcal{C}^{1}$-diffeomorphism which is a minimizer for the variational integral (13.11) subject to given boundary values. Then its inverse $h: \Omega^{\prime} \rightarrow \Omega$ satisfies the Beltrami equation

$$
\frac{\partial h}{\partial \bar{\xi}}=k \frac{\overline{\varphi(\xi)}}{|\varphi(\xi)|} \frac{\partial h}{\partial \xi},
$$

where $\varphi(\xi)=-F^{\prime}(\xi)$ is a holomorphic function in $\Omega^{\prime}$ and $k=k(\xi, h)$ is as in $(13.22)$.

It is appropriate at this stage to recall that mappings satisfying (13.23) with a constant $0 \leqslant k<1$ are referred to as Teichmüller mappings [22, p. 231]. For this reason we shall call $h$ the pseudo-Teichmüller mapping. What is so special about the Beltrami coefficient of $h$ is that its argument is a harmonic function.

Note that in the case when $\Psi(t)=t$ and $h$ is harmonic, as in Theorem 10.8, we find that

$$
\phi(\xi)=h_{\xi} \overline{h_{\bar{\xi}}}
$$

where both $h_{\xi}$ and $\overline{h_{\bar{\xi}}}$ are analytic functions.

The conjugate stationary solutions reduced (13.13) to a first order equation for the inverse. As an alternative development we note that non-divergence forms of equation (13.13) are also interesting. These are actually systems of second order PDEs for the real and imaginary part of $f$. Since the integrand at (13.11) is polyconvex, such systems must satisfy the Legendre-Hadamard, ellipticity condition; see $[\mathbf{4}, \mathbf{1 0}, \mathbf{2 6}]$ for more details. Let us consider the simplest case of 
the distortion function

$$
\mathbb{K}(z, f)=\frac{\|D f(z)\|^{2}}{J(z, f)}=\frac{\left|f_{z}\right|^{2}+\left|f_{\bar{z}}\right|^{2}}{\left|f_{z}\right|^{2}-\left|f_{\bar{z}}\right|^{2}} .
$$

In this case $\Psi(t)=t$ and hence

$$
A(\kappa)=\frac{2\left|f_{z}\right|^{2}\left|f_{\bar{z}}\right|^{2}}{\left(\left|f_{z}\right|^{2}-\left|f_{\bar{z}}\right|^{2}\right)^{2}} .
$$

The Euler-Lagrange equation reads as

$$
\frac{\partial}{\partial z}\left(\frac{\left|f_{z}\right|^{2} f_{\bar{z}}}{J(z, f)^{2}}\right)=\frac{\partial}{\partial \bar{z}}\left(\frac{\left|f_{\bar{z}}\right|^{2} f_{z}}{J(z, f)^{2}}\right) .
$$

Lengthly computation reduces (13.27) to an elegant non-divergence equation

$$
f_{z \bar{z}}=\alpha f_{z z}+\beta f_{\bar{z} \bar{z}}
$$

where

$$
\alpha=\frac{\mu(z, f)}{1+|\mu(z, f)|^{2}}=\frac{\overline{f_{z}} f_{\bar{z}}}{\left|f_{z}\right|^{2}+\left|f_{\bar{z}}\right|^{2}}=\bar{\beta}
$$

see (A.28). This quasilinear system is elliptic, meaning that

$$
|\alpha(z)|+|\beta(z)|=\frac{2 k}{1+k^{2}}<1 .
$$

It is somewhat peculiar that (13.28) turns out to be $\mathbb{C}$-linear with respect to the second order derivatives. We refer to the appendix for a detailed computation.

\subsection{Equations for the inverse map}

Suppose that a $\mathcal{C}^{1}$-diffeomorphism $f: \Omega \rightarrow \Omega^{\prime}$ is a minimizer of the variational integral

$$
\iint_{\Omega} \mathbb{K}_{\Psi}(z, f)|d z|^{2}=\iint_{\Omega} \Psi\left(\frac{\left|f_{z}\right|^{2}+\left|f_{\bar{z}}\right|^{2}}{\left|f_{z}\right|^{2}-\left|f_{\bar{z}}\right|^{2}}\right)|d z|^{2} .
$$

This just amounts to saying that the inverse map $h: \Omega^{\prime} \rightarrow \Omega$ minimizes the integral

$$
\iint_{\Omega^{\prime}} J(\xi, h) \mathbb{K}_{\Psi}(\xi, h)|d \xi|^{2}=\iint_{\Omega^{\prime}}\left(\left|h_{\xi}\right|^{2}-\left|h_{\bar{\xi}}\right|^{2}\right) \Psi\left(\frac{\left|h_{\xi}\right|^{2}+\left|h_{\bar{\xi}}\right|^{2}}{\left|h_{\xi}\right|^{2}-\left|h_{\bar{\xi}}\right|^{2}}\right)|d \xi|^{2} .
$$

This time the variation of the integrand reads as

$$
\left(\dot{J} \Psi+J \Psi^{\prime} \dot{\mathbb{K}}\right)[\eta]=\left(\overline{h_{\xi}} \eta_{\xi}-\overline{h_{\bar{\xi}}} \eta_{\bar{\xi}}\right) \Psi+\frac{2 \kappa J}{(1-\kappa)^{2}}\left(\frac{\eta_{\bar{\xi}}}{h_{\bar{\xi}}}-\frac{\eta_{\xi}}{h_{\xi}}\right) \Psi^{\prime}
$$

whose complex conjugate gives the following divergence form of the LagrangeEuler equation:

$$
\frac{\partial}{\partial \xi}\left(\left[(\mathbb{K}+1) \Psi^{\prime}-\Psi\right] h_{\bar{\xi}}\right)+\frac{\partial}{\partial \bar{\xi}}\left(\left[\Psi-(\mathbb{K}-1) \Psi^{\prime}\right] h_{\xi}\right)=0 .
$$


First we view the square brackets as given measurable coefficients

$$
\frac{\partial}{\partial \xi}\left(M h_{\bar{\xi}}\right)+\frac{\partial}{\partial \bar{\xi}}\left(N h_{\xi}\right)=0,
$$

where

$$
M(\xi)=(\mathbb{K}+1) \Psi^{\prime}(\mathbb{K})-\Psi(\mathbb{K}) \quad \text { and } \quad N(\xi)=\Psi(\mathbb{K})-(\mathbb{K}-1) \Psi^{\prime}(\mathbb{K}),
$$

where $\mathbb{K}=\mathbb{K}(\xi, h)$. From this point of view, if $M=M(\xi)$ and $N=N(\xi)$ happen to be smooth, then the equation (13.35) is elliptic. Indeed,

$$
(M+N) h_{\bar{\xi} \bar{\xi}}+M_{\xi} h_{\bar{\xi}}+N_{\bar{\xi}} h_{\xi}=0, \quad \text { where } M+N=2 \Psi^{\prime}(\mathbb{K})>0 .
$$

On the other hand, we may consider (13.35) as the Lagrange-Euler equation of a quadratic energy integrand

$$
\mathcal{E}[h]=\iint_{\Omega^{\prime}}\left(M\left|h_{\bar{\xi}}\right|^{2}+N\left|h_{\xi}\right|^{2}\right)|d \xi|^{2} .
$$

However, inequality $M+N>0$ is insufficient for this functional to be convex. We must assume that both coefficients $M(\xi)$ and $N(\xi)$ are non-negative. This happens if and only if

$$
\mathbb{K}-1 \leqslant \frac{\Psi(\mathbb{K})}{\Psi^{\prime}(\mathbb{K})} \leqslant \mathbb{K}+1 .
$$

The case $\Psi(\mathbb{K})=\mathbb{K}$ has already been investigated in Theorem 10.12 where we have shown that $h$ then satisfies the Laplace equation. Practically, there are no other examples since (13.39) forces almost linear growth of $\Psi$.

That is why the variational approach has to be abandoned when $\Psi$ has overlinear growth. There is, however, an interesting and promising non-divergence form of (13.34). A tedious computation leads to a second order equation

$$
h_{\xi \bar{\xi}}=\alpha h_{\xi \xi}+\beta h_{\overline{\xi \xi}}+\gamma \overline{h_{\xi \xi}}+\delta \overline{h_{\overline{\xi \xi}}},
$$

where the complex coefficients $\alpha, \beta, \gamma$ and $\delta$ depend in a rather explicit way, by means of $\Psi$, only on the first order derivatives $h_{\xi}$ and $h_{\bar{\xi}}$.

We shall not bother with these explicit formulas here but refer the interested reader to our appendix. What is perhaps more interesting is the ellipticity condition

$$
|1+\alpha(\xi) \lambda+\beta(\xi) \bar{\lambda}|>|\gamma(\xi) \bar{\lambda}+\delta(\xi) \lambda|
$$

for every complex number $\lambda$ of modulus 1 . This means that our second order equation (13.40) is not only elliptic but also lies in the same homotopy class as the Laplacian [1]. Verification of the ellipticity condition at (13.41) is again postponed to the appendix.

\section{Extremal mappings between annuli}

In previous sections we introduced the basic theory for extremal mappings of finite distortion. Here we apply these methods and ideas in the classic setting of identifying extremal mappings of finite distortion between annuli. Here we are quickly brought to consider the properties of harmonic mappings between annuli and the conjecture of Nitsche discussed next. 


\subsection{The Nitsche Conjecture in the plane}

Let $A(\rho, 1)$ be the annulus $\{z \in \mathbb{C}: \rho<|z|<1\}$ where $0<\rho<1$. For a given $\rho$ let $\mathcal{H}(\rho)$ be the family of all harmonic homeomorphisms $h: A(\rho, 1) \rightarrow A(\sigma, 1)$. Denote by $\kappa(\rho)$ the supremum of $\sigma$ as $h$ varies over the family $\mathcal{H}(\rho)$. In 1962 Nitsche showed that $\kappa(\rho)<1$, that is, that $A(\rho, 1)$ cannot be mapped to an arbitrarily thin annulus, and posed a question concerning the validity of the formula

$$
\kappa(\rho)=\frac{2 \rho}{1+\rho^{2}} ;
$$

see [27]. The most recent results towards this conjecture can be found in $[\mathbf{2 4}, \mathbf{3 4}]$. See too the recent monograph $[6]$.

Nitsche's conjecture, together with our earlier results, would have the rather surprising consequence that the $L^{1}$-minimisation problem among the class of homeomorphisms

$$
\mathcal{F}=\left\{f: A(1, \rho) \rightarrow A(1, \sigma) \text { and } f \in W_{\text {loc }}^{1,2}\right\}
$$

has a minimiser if and only if $\sigma \leqslant 2 \rho /\left(1+\rho^{2}\right)$. (Recently the first three authors have completely solved this $L^{1}$-minimisation problem.)

It will be from the point of view of $L^{1}$-minimisers that we consider the problem. We shall also weaken the regularity assumptions to $W^{1,1}$.

\subsection{Extremal mappings between annuli}

Let

$$
A=A(r, R)=\{z \in \mathbb{C}: r<|z|<R\}
$$

denote the usual round annulus in the plane whose modulus is defined to be

$$
\mathcal{M}(A)=\log \frac{R}{r} .
$$

A topological annulus $\mathcal{A}$ is the homeomorphic image of a round annulus. In the plane, this is equivalent to being doubly connected, but this is not so in higher dimensions. We will only be concerned with bounded topological annuli. Such $\mathcal{A}$ can be written as

$$
\mathcal{A}=\Omega \backslash F
$$

where $\Omega$ is a bounded topological disk and $F$ is a relatively compact connected subset.

Consider now the variational problem of minimising the $L^{1}$-norm of the distortion function $\mathbb{K}(z, f)$ among all homeomorphisms $f$ of finite distortion on the annulus $A(r, R)$, normalized so that the modulus of the image has a fixed value $M \leqslant \log (R / r)$. With the next theorem we show that for this problem the minimiser exists, is unique and $C^{\infty}$-smooth. Moreover, the minimiser is a radial function and has the explicit form described in (14.4) and (14.5).

Theorem 14.1. Let $f: A=A(r, R) \rightarrow \mathcal{A}$ be a homeomorphism of finite distortion. Suppose that

$$
\mathcal{M}(\mathcal{A}) \leqslant \log \frac{R}{r}
$$


Then

$$
\iint_{A} \mathbb{K}(z, f)|d z| \geqslant \iint_{A} \mathbb{K}\left(z, f_{0}\right)|d z|,
$$

where the extremal mapping $f_{0}$ has the form

$$
f_{0}\left(\rho e^{i \theta}\right)=e^{i \theta} \varphi(\rho), \quad \text { for } 0 \leqslant \theta<2 \pi, \quad r \leqslant \rho \leqslant R
$$

and

$$
\varphi(t)=t+\sqrt{t^{2}+a^{2}}
$$

and the number $a$ is determined from the equation

$$
\mathcal{M}(\mathcal{A})=2 \log \frac{R+\sqrt{R^{2}+a^{2}}}{r+\sqrt{r^{2}+a^{2}}} .
$$

Equality occurs in (14.3) if and only if $f=\lambda f_{0}+b$ for some $\lambda, b \in \mathbb{C}$, with $\lambda \neq 0$. The extremal map is unique up to conformal change of the target annulus.

Proof. We may assume that $\mathcal{A}=\Omega \backslash F$ is a round annulus with radii

$$
R_{0}=R+\sqrt{R^{2}+a^{2}}, \quad r_{0}=r+\sqrt{r^{2}+a^{2}},
$$

and make a few preliminary observations. First, as $f \in W_{\mathrm{loc}}^{1,1}(A)$ is a homeomorphism, we see that the Jacobian determinant

$$
J(z, f)=\left|f_{z}\right|^{2}-\left|f_{\bar{z}}\right|^{2} \geqslant 0
$$

is an integrable function,

$$
\iint_{A} J(z, f)|d z| \leqslant|\mathcal{A}| .
$$

If, in addition, the distortion function

$$
\mathbb{K}(z, f)=\frac{\left|f_{z}\right|^{2}+\left|f_{\bar{z}}\right|^{2}}{\left|f_{z}\right|^{2}-\left|f_{\bar{z}}\right|^{2}} \in L^{1}(A),
$$

then from Hölder's inequality we see that

$$
\|D f(z)\|=\sqrt{\mathbb{K}(z, f)} \sqrt{J(z, f)} \in L^{1}(A) .
$$

Thus we in fact have $f \in W^{1,1}(A)$.

Let $S_{t}=\{z:|z|=t\}$, with $r<t<R$. Then, as a consequence of Fubini's Theorem, for almost every $t$ the mapping $f \in W^{1,1}\left(S_{t}\right)$ and is absolutely continuous. For such $t$, the image of the circle $S_{t}$ is a rectifiable Jordan curve for which we have the length inequality

$$
\left|f\left(S_{t}\right)\right| \leqslant \int_{|z|=t}\left(\left|f_{z}\right|+\left|f_{\bar{z}}\right|\right)|d z| .
$$

.Next, an elementary computation provides us with the identity

$$
\left|f_{z}\right|+\left|f_{\bar{z}}\right|=\left(\sqrt{\frac{\mathbb{K}+1}{2}}+\sqrt{\frac{\mathbb{K}-1}{2}}\right) \sqrt{J(z, f)}
$$


with $\mathbb{K}=\mathbb{K}(z, f)$. We now apply Hölder's inequality to see that

$$
\left(\int_{S_{t}}\left|f_{z}\right|+\left|f_{\bar{z}}\right|\right)^{2} \leqslant\left(\int_{S_{t}} \mathbb{K}+\sqrt{\mathbb{K}^{2}-1}\right) \int_{S_{t}} J(z, f) .
$$

The function $x \mapsto x+\sqrt{x^{2}-1}$ is concave for $x \geqslant 1$ and so we may apply Jensens' inequality to see that

$$
\begin{aligned}
\int_{S_{t}} \mathbb{K}+\sqrt{\mathbb{K}^{2}-1} & \leqslant 2 \pi t\left(f_{S_{t}} \mathbb{K}+\sqrt{\left(f_{S_{t}} \mathbb{K}\right)^{2}-1}\right) \\
& =: \frac{2 \pi t}{g(t)}
\end{aligned}
$$

Then of course

$$
\frac{1}{2 \pi t} \int_{S_{t}} \mathbb{K}(z, f)=\frac{1}{2}\left(g(t)+\frac{1}{g(t)}\right) .
$$

If we substitute this back into (14.8), we find that

$$
\left(\int_{S_{t}}\left|f_{z}\right|+\left|f_{\bar{z}}\right|\right)^{2} \leqslant \frac{2 \pi t}{g(t)} \int_{S_{t}} J(z, f) .
$$

Now we put this into the isoperimetric inequality

$$
\begin{aligned}
|F|+\iint_{r<|z|<t} J(z, f) & \leqslant \frac{1}{4 \pi}\left(\int_{S_{t}}\left|f_{z}\right|+\left|f_{\bar{z}}\right|\right)^{2} \\
& \leqslant \frac{t}{2 g(t)} \int_{S_{t}} J(z, f) .
\end{aligned}
$$

Equivalently,

$$
\frac{\int_{S_{t}} J(z, f)}{|F|+\iint_{r<|z|<t} J(z, f)} \geqslant \frac{2 g(t)}{t}
$$

Once we notice that the numerator is the derivative of the denominator on the left-hand side of (14.10) we can integrate this inequality to obtain

$$
\log \left(1+\frac{1}{|F|} \iint_{r<|z|<R} J(z, f)\right) \geqslant 2 \int_{r}^{R} \frac{g(t)}{t} d t .
$$

Here the area inequality $|F|+\iint_{r<|z|<t} J(z, f) \leqslant \pi R_{0}^{2}$ simplifies this expression to

$$
\int_{r}^{R} \frac{g(t)}{t} d t \leqslant M=\log \frac{R_{0}}{r_{0}} .
$$

The next step we wish to take is to compare the above computation with the particular case $f=f_{0}$. There we see that

$$
\begin{aligned}
g_{0}(t) & =t / \sqrt{t^{2}+a^{2}} \\
\mathbb{K}_{0}(z, f) & =\frac{1}{2}\left(g_{0}(|z|)+\frac{1}{g_{0}(|z|)}\right), \\
\int_{r}^{R} \frac{g_{0}(t)}{t} d t & =\log \frac{R_{0}}{r_{0}} .
\end{aligned}
$$


This tells us that

$$
\int_{r}^{R} \frac{g(t)-g_{0}(t)}{t} d t \leqslant 0
$$

while $1-1 / g_{0}^{2}(t)=-a^{2} / t^{2}$. Further, the convexity of $g \mapsto \frac{1}{2}(g+1 / g)$ implies that

$$
\begin{aligned}
\left(g+\frac{1}{g}\right) & \geqslant\left(g_{0}+\frac{1}{g_{0}}\right)+\left(1-\frac{1}{g_{0}^{2}}\right)\left(g-g_{0}\right) \\
& =\left(g_{0}+\frac{1}{g_{0}}\right)-\frac{a^{2}}{t^{2}}\left(g-g_{0}\right) .
\end{aligned}
$$

To complete the argument, we note the inequality

$$
\begin{aligned}
\iint_{A} \mathbb{K}(z, f) & =\int_{r}^{R} \int_{S_{t}} \mathbb{K}(z, f) d t \\
& =\pi \int_{r}^{R}\left(g+\frac{1}{g}\right) t d t \\
& \geqslant \pi \int_{r}^{R}\left(g_{0}+\frac{1}{g_{0}}\right) t d t-\pi a^{2} \int_{r}^{R} \frac{g-g_{0}}{t} d t \\
& \geqslant \iint_{A} \mathbb{K}\left(z, f_{0}\right) .
\end{aligned}
$$

Tracing the argument back, we see that equality holds only when $f$ is obtained from $f_{0}$ by a rotation.

\section{Appendix}

\section{A.1. More about distortion functions}

For a non-singular matrix $A \in \mathbb{R}_{+}^{n \times n}$ we obtain, from (3.7) and (3.8),

$$
\sqrt[\ell]{\mathbb{K}_{\ell}\left(A^{-1}\right)}=\sqrt[[n-\ell]]{\mathbb{K}_{n-\ell}(A)}
$$

Various bounds of norms of the matrices $A^{\ell \times \ell}$ will be useful.

Lemma A.1 (Hadamard-type inequality). For every $1 \leqslant \ell \leqslant \kappa \leqslant n$ and $A \in \mathbb{R}^{n \times n}$, we have

$$
\left|A^{\kappa \times \kappa}\right|^{1 / \kappa} \leqslant\left|A^{\ell \times \ell}\right|^{1 / \ell} \text { and }\left\|A^{\kappa \times \kappa}\right\|^{1 / \kappa} \leqslant\left\|A^{\ell \times \ell}\right\|^{1 / \ell} .
$$

Proof. It involves no loss of generality to assume that $A$ is diagonal, say $A=\operatorname{diag}\left\{\lambda_{1}, \ldots, \lambda_{n}\right\}$, where $0<\lambda_{1} \leqslant \ldots \leqslant \lambda_{n}$. The first inequality reduces, equivalently, to

$$
\left(\lambda_{n} \lambda_{n-1} \ldots \lambda_{n-\kappa+1}\right)^{1 / \kappa} \leqslant\left(\lambda_{n} \lambda_{n-1} \ldots \lambda_{n-\ell+1}\right)^{1 / \ell}
$$

which is easy to verify. The second inequality has already been pointed out in $[\mathbf{1 5}$, Lemma 2.1] with a proof based on symmetric averages; see also [25].

Hadamard's inequalities give sharp relations between the distortion functions. 
LEMma A.2. We have the following chains of inequalities:

$$
K_{1} \leqslant K_{2} \leqslant \ldots \leqslant K_{\ell} \leqslant \ldots \leqslant K_{n-2} \leqslant K_{n-1}
$$

and

$$
K_{1}^{n-1} \geqslant K_{2}^{(n-2) / 2} \geqslant \ldots \geqslant K_{\ell}^{(n-\ell) / \ell} \geqslant \ldots \geqslant K_{n-2}^{2 /(n-2)} \geqslant K_{n-1}^{1 /(n-1)} .
$$

Similarly,

$$
\mathbb{K}_{1} \leqslant \mathbb{K}_{2} \leqslant \ldots \leqslant \mathbb{K}_{\ell} \leqslant \ldots \leqslant \mathbb{K}_{n-2} \leqslant \mathbb{K}_{n-1}
$$

and

$$
\mathbb{K}_{1}^{n-1} \geqslant \mathbb{K}_{2}^{(n-2) / 2} \geqslant \ldots \geqslant \mathbb{K}_{\ell}^{(n-\ell) / \ell} \geqslant \ldots \geqslant \mathbb{K}_{n-2}^{2 /(n-2)} \geqslant \mathbb{K}_{n-1}^{1 /(n-1)}
$$

Proof. Let $A \in \mathbb{R}_{+}^{n \times n}$. We may assume that $\operatorname{det} A=1$. For (A.5) we apply Lemma A.1. Given $1 \leqslant \ell \leqslant \kappa \leqslant n$, we have

$$
\left[K_{\ell}(A)\right]^{(n-\ell) / \ell}=\left|A^{\ell \times \ell}\right|^{n / \ell} \geqslant\left|A^{\kappa \times \kappa}\right|^{n / \kappa}=\left[K_{\kappa}(A)\right]^{(n-\kappa) / \kappa},
$$

as desired. The same arguments give inequalities at (A.7) where, instead of the operator norm, the mean Hilbert-Schmidt norm has been used in the definition of the distortion functions.

For the inequality at (A.4) we argue in much the same way. This time, we express $K_{\ell}$ in terms of the inverse matrix. Since $\operatorname{det} A=1$, we have

$$
\begin{aligned}
K_{\ell}(A) & =\left[K_{n-\ell}\left(A^{-1}\right)\right]^{\ell /(n-\ell)}=\left|\left[A^{-1}\right]^{(n-\ell) \times(n-\ell)}\right|^{n /(n-\ell)} \\
& \leqslant\left|\left[A^{-1}\right]^{(n-\kappa) \times(n-\kappa)}\right|^{n /(n-\kappa)}=\left|A^{\kappa \times \kappa}\right|^{n /(n-\kappa)}=K_{\kappa}(A)
\end{aligned}
$$

by (3.7) and (3.8). Again, the same arguments give the inequalities at (A.6), completing the proof.

\section{A.2. Polyconvexity}

A matrix function $\Xi: \mathbb{R}^{n \times n} \rightarrow \mathbb{R}$ is said to be polyconvex if it can be written as

$$
\Xi=F\left(A^{1 \times 1}, A^{2 \times 2}, \ldots, A^{n \times n}\right)
$$

where $\left(A^{1 \times 1}, A^{2 \times 2}, \ldots, A^{n \times n}\right)$ is a list of all possible minors of $A \in \mathbb{R}^{n \times n}$ and the function $F$ is convex. The list of minors can be identified with a point in $\mathbb{R}^{N}$, where

$$
N=\left(\begin{array}{l}
n \\
1
\end{array}\right)^{2}+\left(\begin{array}{l}
n \\
2
\end{array}\right)^{2}+\ldots+\left(\begin{array}{l}
n \\
n
\end{array}\right)^{2}=\frac{(2 n) !}{n ! n !}-1 .
$$

Thus $F: \mathbb{R}^{N} \rightarrow \mathbb{R}$.

Our basic examples of polyconvex functions are the distortion functions

$$
\mathbb{K}_{1}, \ldots, \mathbb{K}_{n-1}: \mathbb{R}_{+}^{n \times n} \rightarrow \mathbb{R}
$$

Precisely,

$$
\mathbb{K}_{\ell}(A)=\frac{\left\|A^{\ell \times \ell}\right\|^{n /(n-\ell)}}{(\operatorname{det} A)^{\ell /(n-\ell)}}
$$

is a convex function of $\operatorname{det} A$ and the $\ell$-minors. For this, we observe that the function $x^{\alpha} / y^{\beta}$ of two variables $x, y \in \mathbb{R}_{+}$is convex whenever $\alpha \geqslant \beta+1 \geqslant 1$; see 
[16, Lemma 8.8.2]. In particular,

$$
\frac{x^{\alpha}}{y^{\beta}}-\frac{a^{\alpha}}{b^{\beta}} \geqslant \alpha \frac{a^{\alpha-1}}{b^{\beta}}(x-a)-\beta \frac{a^{\alpha}}{b^{\beta+1}}(y-b) .
$$

Hence,

$$
\begin{aligned}
\mathbb{K}_{\ell}(X)-\mathbb{K}_{\ell}(A) \geqslant & \frac{n}{n-\ell}\left(\frac{\left\|A^{\ell \times \ell}\right\|}{\operatorname{det} A}\right)^{\ell /(n-\ell)}\left(\|X\|^{\ell \times \ell}-\left\|A^{\ell \times \ell}\right\|\right) \\
& -\frac{\ell}{n-\ell}\left(\frac{\left\|A^{\ell \times \ell}\right\|}{\operatorname{det} A}\right)^{n /(n-\ell)}(\operatorname{det} X-\operatorname{det} A) \\
\geqslant & \left(\begin{array}{c}
n-1 \\
\ell
\end{array}\right)^{-1}\left(\frac{\left\|A^{\ell \times \ell}\right\|}{\operatorname{det} A}\right)^{\ell /(n-\ell)}\left\langle\frac{A^{\ell \times \ell}}{\left\|A^{\ell \times \ell}\right\|}, X^{\ell \times \ell}-A^{\ell \times \ell}\right\rangle \\
& -\frac{\ell}{n-\ell}\left(\frac{\left\|A^{\ell \times \ell}\right\|}{\operatorname{det} A}\right)^{n /(n-\ell)}(\operatorname{det} X-\operatorname{det} A) .
\end{aligned}
$$

More generally,

$$
P_{\mathbb{K}}(A)=P\left(\mathbb{K}_{1}(A), \ldots, \mathbb{K}_{n-1}(A)\right): \mathbb{R}_{+}^{n \times n} \rightarrow[1, \infty),
$$

where

$$
P: \underbrace{[1, \infty) \times \ldots \times[1, \infty)}_{(n-1) \text { times }} \rightarrow[1, \infty)
$$

is a given convex function non-decreasing in each variable, when all the other variables are held fixed. That is, the partials $P_{i}=\partial P / \partial_{i}$ are non-negative. In particular, we have

$$
\begin{aligned}
P_{\mathbb{K}}(X)-P_{\mathbb{K}}(A) & \geqslant \sum_{\ell=1}^{n-1} \Pi_{\ell}(A)\left[\mathbb{K}_{\ell}(X)-\mathbb{K}_{\ell}(A)\right] \\
& \geqslant \sum_{\ell=1}^{n}\left\langle G_{\ell}(A), X^{\ell \times \ell}-A^{\ell \times \ell}\right\rangle .
\end{aligned}
$$

\section{A.3. Second order elliptic system}

The variational equations are very useful in the study of extremal problems. Both the extremal mapping and its inverse turn out to satisfy their own second order system of PDEs. It is important to examine the ellipticity of such systems. We include in this appendix a brief discussion of the systems of two equations with two unknowns. Using complex numbers we find that the system reduces to one complex equation. The general form of the second order elliptic operator is

$$
\mathcal{L}=\mathfrak{a}(z) \frac{\partial^{2}}{\partial z \partial \bar{z}}+\mathfrak{b}(z) \frac{\partial^{2}}{\partial z \partial z}+\mathfrak{c}(z) \frac{\partial^{2}}{\partial \bar{z} \partial \bar{z}}+\mathfrak{d}(z) \frac{\overline{\partial^{2}}}{\partial z \partial \bar{z}}+\mathfrak{e}(z) \overline{\frac{\partial^{2}}{\partial z \partial z}}+\mathfrak{f}(z) \frac{\overline{\partial^{2}}}{\partial \bar{z} \partial \bar{z}} .
$$

For a fixed $z \in \Omega \subset \mathbb{C}$, the coefficients give rise to a point $(\mathfrak{a}, \mathfrak{b}, \mathfrak{c}, \mathfrak{d}, \mathfrak{e}, \mathfrak{f}) \in \mathbb{C}^{6}$. The ellipticity conditions determine an open set $\mathbb{E} \subset \mathbb{C}^{6}$. This set, as shown by Bojarski 
[1], consists of six components represented by

$$
\begin{cases}\frac{\partial^{2}}{\partial z \partial \bar{z}}, & \overline{\frac{\partial^{2}}{\partial z \partial \bar{z}}} \\ \frac{\partial^{2}}{\partial z \partial z}, & \frac{\partial^{2}}{\partial z \partial z}, \\ \frac{\partial^{2}}{\partial \bar{z} \partial \bar{z}}, & \frac{\partial^{2}}{\partial \bar{z} \partial \bar{z}},\end{cases}
$$

In contrast with the scalar elliptic equations the elliptic systems with measurable coefficients are not well developed yet; see $[\mathbf{1 3}, \mathbf{9}]$ for details. It turns out that the systems in the same homotopy class as the Laplacian $\triangle=4 \partial^{2} / \partial z \partial \bar{z}$ (or its complex conjugate $\bar{\triangle}$ ) are best suited to the regularity properties of the solutions. In $[\mathbf{1}]$ it is shown that such systems take the form

$$
f_{z \bar{z}}=\alpha(z) f_{z z}+\beta(z) f_{\bar{z} \bar{z}}+\gamma(z) \overline{f_{z z}}+\delta(z) \overline{f_{\bar{z} \bar{z}}},
$$

where the ellipticity condition reads as

$$
|1+\alpha(z) \lambda+\beta(z) \bar{\lambda}|>|\gamma(z) \bar{\lambda}+\delta(z) \lambda|
$$

for all $\lambda \in \mathbb{C}$ with modulus 1 . The next two subsections are devoted to a computation showing that variational equations for the extremal mappings belong to this homotopy class.

A.3.1. The Lagrange-Euler equation in non-divergence form. We begin this section by issuing a warning. The computations below can only be rigorously justified for specific extremals only after we have established sufficient regularity.

We are going to express the variational equation (13.13) in non-divergence form. In our computation the explicit formula for $A=A(\kappa)$ is irrelevant. Before formulating the equation we need to introduce the following coefficients:

$$
a(z)=\frac{f_{z}}{\left|f_{z}\right|}, \quad b(z)=\frac{f_{\bar{z}}}{\left|f_{\bar{z}}\right|}
$$

Hence, the complex dilatation of $f$ is

$$
\mu(z)=\frac{f_{\bar{z}}}{f_{z}}=\bar{a} b k
$$

where $k=\left|f_{\bar{z}}\right| /\left|f_{z}\right|$. 
Lemma A.3. Equation (13.13) is equivalent to

$$
\begin{aligned}
(1-\kappa) \frac{\kappa A^{\prime}}{A} \frac{\partial^{2} f}{\partial z \partial \bar{z}}= & \mu\left(\frac{\kappa A^{\prime}}{A}-\frac{2 \kappa}{1-\kappa}\right) \frac{\partial^{2} f}{\partial z \partial z} \\
& +\bar{\mu}\left(\frac{2}{1-\kappa}-\frac{\kappa A^{\prime}}{A}\right) \frac{\partial^{2} f}{\partial \bar{z} \partial \bar{z}} \\
& +a^{2} \kappa\left(\frac{\kappa A^{\prime}}{A}-\frac{1+\kappa)}{1-\kappa}\right) \frac{\overline{\partial^{2} f}}{\partial z \partial z} \\
& +b^{2}\left(\frac{1+\kappa}{1-\kappa}-\frac{\kappa A^{\prime}}{A}\right) \frac{\overline{\partial^{2} f}}{\partial \bar{z} \partial \bar{z}}
\end{aligned}
$$

Proof. For notational simplicity we introduce the function $\mathcal{A}(k)=A\left(k^{2}\right)=A(\kappa)$ and collect some formulas first. The chain rule gives

$$
\begin{aligned}
& \frac{\partial \mathcal{A}}{\partial z}=\mathcal{A}^{\prime}(k) \frac{\partial k}{\partial z}, \\
& \frac{\partial \mathcal{A}}{\partial \bar{z}}=\mathcal{A}^{\prime}(k) \frac{\partial k}{\partial \bar{z}} .
\end{aligned}
$$

The derivatives of $k$ become linear forms of the second derivatives of $f$, namely

$$
\begin{aligned}
& 2\left|f_{z}\right| \frac{\partial k}{\partial z}=b \overline{f_{\bar{z} \bar{z}}}+\bar{b} f_{z \bar{z}}-k a \overline{f_{z \bar{z}}}-k \bar{a} f_{z z}, \\
& 2\left|f_{z}\right| \frac{\partial k}{\partial \bar{z}}=\bar{b} f_{\bar{z} \bar{z}}+b \overline{f_{z \bar{z}}}-k \bar{a} f_{z \bar{z}}-k a \overline{f_{z z}} .
\end{aligned}
$$

Equation (13.13) expands into

$$
\left(f_{\bar{z}} f_{z}^{2} k_{\bar{z}}-f_{z} f_{\bar{z}}^{2} k_{z}\right) \mathcal{A}^{\prime}(k)=\left(f_{z}^{2} f_{\bar{z} \bar{z}}-f_{\bar{z}}^{2} f_{z z}\right) \mathcal{A}(k)
$$

which, in view of formulas (A.23) and (A.24), takes the form

$$
\begin{aligned}
-2 k f_{z \bar{z}}+\left(1+k^{2}\right) a b \overline{f_{z \bar{z}}} & +k^{2} \bar{a} b\left(1+\frac{2 \mathcal{A}}{k \mathcal{A}^{\prime}}\right) f_{z z}-k a^{2} \overline{f_{z z}} \\
& +a \bar{b}\left(1-\frac{2 \mathcal{A}}{k \mathcal{A}^{\prime}}\right) f_{\overline{z z}}-k b^{2} \overline{f_{\overline{z z}}}=0
\end{aligned}
$$

Finally, we solve this linear equation for $f_{z \bar{z}}$ in terms of $f_{z z}$ and $f_{\overline{z z}}$ to conclude with (A.20).

It is interesting to know when the equation (A.20) is linear over the field of complex numbers. This happens if and only if

$$
\frac{\kappa A^{\prime}(\kappa)}{A(\kappa)}=\frac{1+\kappa}{1-\kappa}=\mathbb{K}(z, f),
$$

in which case the equation reduces to

$$
(1+\kappa) f_{z \bar{z}}=\mu f_{z z}+\bar{\mu} f_{\overline{z z}}
$$

as claimed at (13.28). Note that the only solution to (A.27), up to a multiplicative 
constant, is

$$
A(\kappa)=\frac{2 \kappa}{(1-\kappa)^{2}}
$$

as in (13.26).

Recall that the formula (A.20) is the variational equation for the functional

$$
\iint_{\Omega} \mathbb{K}_{\Psi}(z, f)|d z|^{2}
$$

where

$$
\mathbb{K}_{\Psi}=\Psi(\mathbb{K})=\Psi\left(\frac{1+\kappa}{1-\kappa}\right)
$$

and $\Psi:[1, \infty) \rightarrow[1, \infty)$ is convex, with $\Psi(1)=1$. In this case

$$
A(\kappa)=\Psi^{\prime}\left(\frac{1+\kappa}{1-\kappa}\right) \frac{2 \kappa}{(1-\kappa)^{2}} .
$$

We want hence to express $\kappa A^{\prime}(\kappa) / A(\kappa)$ in terms of $\Psi$. Elementary computation yields

$$
\frac{2 \kappa A^{\prime}(\kappa)}{A(\kappa)}=2 \mathbb{K}+\left(\mathbb{K}^{2}-1\right) \frac{\Psi^{\prime \prime}}{\Psi^{\prime}} .
$$

On substituting this into (A.20) we obtain

$$
\begin{aligned}
2\left[\frac{2 \mathbb{K} \Psi^{\prime}}{\mathbb{K}+1}+(\mathbb{K}-1) \Psi^{\prime \prime}\right] \frac{\partial^{2} f}{\partial z \partial \bar{z}}= & {\left[2 \Psi^{\prime}+\left(\mathbb{K}^{2}-1\right) \Psi^{\prime \prime}\right] \frac{f_{\bar{z}}}{f_{z}} \frac{\partial^{2} f}{\partial z \partial z} } \\
& +\left[2 \Psi^{\prime}-\left(\mathbb{K}^{2}-1\right) \Psi^{\prime \prime}\right] \frac{\overline{f_{\bar{z}}}}{\overline{f_{z}}} \frac{\partial^{2} f}{\partial \bar{z} \partial \bar{z}} \\
& +\left[(\mathbb{K}-1)^{2} \Psi^{\prime \prime}\right] \frac{f_{z}}{\overline{f_{z}}} \frac{\overline{\partial^{2} f}}{\partial z \partial z} \\
& -\left[\left(\mathbb{K}^{2}-1\right) \Psi^{\prime \prime}\right] \frac{f_{\bar{z}}}{\overline{f_{\bar{z}}}} \frac{\partial^{2} f}{\partial \bar{z} \partial \bar{z}} .
\end{aligned}
$$

A.3.2. Non-divergence equation for the inverse map. We begin with the system in divergence form

$$
\left(\left[\Psi(\mathbb{K})-(\mathbb{K}+1) \Psi^{\prime}(\mathbb{K})\right] h_{\bar{\zeta}}\right)_{\zeta}=\left(\left[\Psi(\mathbb{K})-(\mathbb{K}-1) \Psi^{\prime}(\mathbb{K})\right] h_{\zeta}\right)_{\bar{\zeta}}
$$

which we have derived at (13.34). To make further calculations we need the following identity:

$$
2\left|h_{\zeta}\right|^{2} \mathbb{K}_{\zeta}=(\mathbb{K}+1)^{2}\left[h_{\overline{\zeta \zeta}} \overline{h_{\bar{\zeta}}}+\overline{h_{\overline{\zeta \zeta}}} h_{\bar{\zeta}}\right]-(\mathbb{K}-1)^{2}\left[h_{\zeta \zeta} \overline{h_{\zeta}}+\overline{h_{\overline{\zeta \zeta}}} h_{\zeta}\right]
$$

As $\mathbb{K}=(1+\kappa) /(1-\kappa)$, we find that

$$
\mathbb{K}_{\zeta}=\frac{2}{(1-\kappa)^{2}} \frac{\partial \kappa}{\partial \zeta}
$$


and we compute

$$
\left|h_{\zeta}\right|^{2} \frac{\partial \kappa}{\partial \zeta}=\left|h_{\zeta}\right|^{2}\left(\frac{\left|h_{\bar{\zeta}}\right|^{2}}{\left|h_{\zeta}\right|^{2}}\right)_{\zeta}=\left(h_{\overline{\zeta \zeta}} \overline{h_{\bar{\zeta}}}+\overline{h_{\overline{\zeta \zeta}}} h_{\bar{\zeta}}\right)-\kappa\left(h_{\zeta \zeta} \overline{h_{\zeta}}+\overline{h_{\overline{\zeta \zeta}}} h_{\zeta}\right)
$$

from which formula (A.36) can be obtained.

Now, formally differentiating the equations in divergence form gives us the second order system

$$
\begin{aligned}
0=4 \Psi^{\prime}(\mathbb{K})\left|h_{\zeta}\right|^{2} h_{\overline{\zeta \zeta}} & +(\mathbb{K}+1)^{3}\left(h_{\overline{\zeta \zeta}}\left|h_{\bar{\zeta}}\right|^{2}+\overline{h_{\overline{\zeta \zeta}}} h_{\bar{\zeta}}^{2}\right) \Psi^{\prime \prime}(\mathbb{K}) \\
& -(\mathbb{K}+1)(\mathbb{K}-1)^{2}\left(h_{\zeta \zeta} h_{\bar{\zeta}} \overline{h_{\zeta}}+\overline{h_{\zeta \bar{\zeta}}} h_{\bar{\zeta}} h_{\zeta}\right) \Psi^{\prime \prime}(\mathbb{K}) \\
& -(\mathbb{K}-1)(\mathbb{K}+1)^{2}\left(\overline{h_{\overline{\zeta \zeta}}} h_{\bar{\zeta}} h_{\zeta}+h_{\overline{\zeta \zeta}} \overline{h_{\bar{\zeta}}} h_{\zeta}\right) \Psi^{\prime \prime}(\mathbb{K}) \\
& +(\mathbb{K}-1)^{3}\left(\overline{h_{\zeta \zeta}} h_{\zeta}^{2}+h_{\bar{\zeta}}\left|h_{\zeta}\right|^{2}\right) \Psi^{\prime \prime}(\mathbb{K})
\end{aligned}
$$

We write $\Phi(\mathbb{K})=\Psi^{\prime}(\mathbb{K}) / \Psi^{\prime \prime}(\mathbb{K})$ and collect terms to find that

$$
\begin{aligned}
0= & h_{\overline{\zeta \zeta}}\left[4\left|h_{\zeta}\right|^{2} \Phi+(\mathbb{K}+1)^{3}\left|h_{\bar{\zeta}}\right|^{2}+(\mathbb{K}-1)^{3}\left|h_{\zeta}\right|^{2}\right] \\
& -\overline{h_{\overline{\zeta \zeta}}}\left[(\mathbb{K}+1)(\mathbb{K}-1)^{2} h_{\bar{\zeta}} h_{\zeta}+(\mathbb{K}-1)(\mathbb{K}+1)^{2} h_{\bar{\zeta}} h_{\zeta}\right] \\
& -h_{\zeta \zeta}\left[(\mathbb{K}+1)(\mathbb{K}-1)^{2} h_{\bar{\zeta}} \overline{h_{\zeta}}\right] \\
& +\overline{h_{\zeta \zeta}}\left[(\mathbb{K}-1)^{3} h_{\zeta}^{2}\right] \\
& +\overline{h_{\overline{\zeta \zeta}}}\left[(\mathbb{K}+1)^{3} h_{\bar{\zeta}}^{2}\right] \\
& -h_{\overline{\zeta \zeta}}\left[(\mathbb{K}-1)(\mathbb{K}+1)^{2} \overline{h_{\bar{\zeta}}} h_{\zeta}\right] .
\end{aligned}
$$

and

$$
\begin{aligned}
0= & h_{\overline{\zeta \zeta}}\left[4 \Phi+(\mathbb{K}+1)^{3} \kappa+(\mathbb{K}-1)^{3}\right] \\
& -\overline{h_{\overline{\zeta \zeta}}}\left[(\mathbb{K}+1)(\mathbb{K}-1)^{2}+(\mathbb{K}-1)(\mathbb{K}+1)^{2}\right] \frac{h_{\bar{\zeta}}}{\overline{h_{\zeta}}} \\
& -h_{\zeta \zeta}\left[(\mathbb{K}+1)(\mathbb{K}-1)^{2}\right] \frac{h_{\bar{\zeta}}}{h_{\zeta}} \\
& +\overline{h_{\zeta \zeta}}\left[(\mathbb{K}-1)^{3}\right] \frac{h_{\zeta}}{\overline{h_{\zeta}}} \\
& +\overline{h_{\overline{\zeta \zeta}}}\left[(\mathbb{K}+1)^{3}\right] \frac{h_{\bar{\zeta}}^{2}}{\left|h_{\zeta}\right|^{2}} \\
& -h_{\overline{\zeta \zeta}}\left[(\mathbb{K}-1)(\mathbb{K}+1)^{2}\right] \frac{\overline{h_{\bar{\zeta}}}}{\overline{h_{\zeta}}}
\end{aligned}
$$


We simplify these terms and remove the common factor of $\mathbb{K}-1$ :

$$
\begin{aligned}
0= & h_{\zeta \bar{\zeta}}\left[\frac{4 \Phi}{\mathbb{K}-1}+2\left(\mathbb{K}^{2}+1\right)\right]-\overline{h_{\bar{\zeta}}}[2 \mathbb{K}(\mathbb{K}+1)] \frac{h_{\bar{\zeta}}}{\overline{h_{\zeta}}} \\
& -h_{\zeta \zeta}[(\mathbb{K}+1)(\mathbb{K}-1)] \frac{h_{\bar{\zeta}}}{h_{\zeta}} \overline{\overline{h_{\zeta \zeta}}}\left[(\mathbb{K}-1)^{2}\right] \frac{h_{\zeta}}{\overline{h_{\zeta}}} \\
& +\overline{h_{\overline{\zeta \zeta}}}\left[(\mathbb{K}+1)^{2}\right] \frac{h_{\bar{\zeta}}^{2}}{\left|h_{\bar{\zeta}}\right|^{2}}-h_{\overline{\zeta \zeta}}\left[(\mathbb{K}+1)^{2}\right] \frac{\overline{h_{\bar{\zeta}}}}{\overline{h_{\zeta}}}
\end{aligned}
$$

Now to simplify matters we make a couple of substitutions and write everything in terms of $k=k(\zeta, h)$; here recall that we have used the notation $\kappa=k^{2}$ above. We put

and recall that

$$
a=\frac{h_{\zeta}}{\left|h_{\zeta}\right|}, \quad b=\frac{h_{\bar{\zeta}}}{\left|h_{\bar{\zeta}}\right|}
$$

$$
\mathbb{K}=\frac{1+k^{2}}{1-k^{2}}, \quad \text { whence } \quad \mathbb{K}+1=\frac{2}{1-k^{2}} \quad \text { and } \quad \mathbb{K}-1=\frac{2 k^{2}}{1-k^{2}} .
$$

Our equations now have a common factor $4\left(1-k^{2}\right)^{2}$ and when this is removed and we substitute

they read as

$$
P=\frac{2\left(1-k^{2}\right)^{2} \Phi}{k^{2}}=\frac{2\left(1-k^{2}\right)^{2} \Psi^{\prime}}{k^{2} \Psi^{\prime \prime}}
$$

$$
\begin{aligned}
0= & {\left[P+\left(1+k^{4}\right)\right] h_{\overline{\zeta \zeta}}-\left(1+k^{2}\right) k a b \overline{h_{\overline{\zeta \zeta}}}-k^{3} \bar{a} b h_{\zeta \zeta} } \\
& +k^{4} a^{2} \overline{h_{\zeta \zeta}}+b^{2} \overline{h_{\overline{\zeta \zeta}}}-k a \bar{b} h_{\overline{\zeta \zeta}} .
\end{aligned}
$$

The complex conjugate equation reads as

$$
\begin{aligned}
0= & {\left[P+\left(1+k^{4}\right)\right] \overline{h_{\overline{\zeta \zeta}}}-\left(1+k^{2}\right) k \overline{a b} h_{\zeta \bar{\zeta}}-k^{3} \bar{b} a \overline{h_{\zeta \zeta}} } \\
& +k^{4} \bar{a}^{2} h_{\zeta \zeta}+\bar{b}^{2} h_{\overline{\zeta \zeta}}-k b \bar{a} \overline{h_{\overline{\zeta \zeta}}} .
\end{aligned}
$$

We multiply (A.37) by $\left[P+\left(1+k^{4}\right)\right]$ and equation (A.38) by $\left(1+k^{2}\right) k a b$ and add

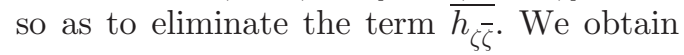

$$
\begin{aligned}
0= & \left.h_{\zeta \bar{\zeta}}\left[P+\left(1+k^{4}\right)\right]^{2}-\left(1+k^{2}\right)^{2} k^{2}\right] \\
& +h_{\zeta \zeta}\left[-P-\left(1+k^{4}\right)+k^{2}\left(1+k^{2}\right)\right] k^{3} \bar{a} b \\
& +\overline{h_{\zeta \zeta}}\left[P+\left(1+k^{4}\right)-\left(1+k^{2}\right)\right] k^{4} a^{2} \\
& +\overline{h_{\overline{\zeta \zeta}}}\left[P+\left(1+k^{4}\right)-\left(1+k^{2}\right) k^{2}\right] b^{2} \\
& +h_{\overline{\zeta \zeta}}\left[-P-\left(1+k^{4}\right)+\left(1+k^{2}\right)\right] k a \bar{b},
\end{aligned}
$$

which simplifies to the following result.

Lemma A.4. The inverse mapping satisfies the equation

$$
\begin{aligned}
{\left[P^{2}+\right.} & \left.2 P\left(1+k^{4}\right)+\left(1-k^{2}\right)\left(1-k^{6}\right)\right] h_{\overline{\zeta \zeta}} \\
= & {\left.\left[P+1-k^{2}\right)\right] k^{3} \bar{a} b h_{\zeta \zeta}+\left[P-k^{2}\left(1-k^{2}\right)\right] k a \bar{b} h_{\overline{\zeta \zeta}} } \\
& +\left[k^{2}\left(1-k^{2}\right)-P\right] k^{4} a^{2} \overline{h_{\zeta \zeta}}+\left[k^{2}-P-1\right] b^{2} \overline{h_{\overline{\zeta \zeta}}}
\end{aligned}
$$


where

$$
P=\frac{2\left(1-k^{2}\right)^{2} \Psi^{\prime}}{k^{2} \Psi^{\prime \prime}}
$$

In a moment we are going to try to determine if this system is elliptic and in order to do this we need to simplify these equations as much as possible. For this reason we introduce the new variable

$$
D=\frac{2 \Psi^{\prime}(\mathbb{K})}{\left(\mathbb{K}^{2}-1\right) \Psi^{\prime \prime}(\mathbb{K})}
$$

and remove a further common factor to obtain the system

$$
\begin{aligned}
0= & {\left[D^{2}\left(1-k^{2}\right)+2 D\left(1+k^{4}\right)+\left(1-k^{6}\right)\right] h_{\zeta \bar{\zeta}} } \\
& +[D+1] k^{3} \bar{a} b h_{\zeta \zeta}-\left[k^{2}-D\right] k a \bar{b} h_{\overline{\zeta \zeta}} \\
& +\left[D-k^{2}\right] k^{4} a^{2} \overline{h_{\zeta \zeta}}+[D+1] b^{2} \overline{h_{\overline{\zeta \zeta}}}
\end{aligned}
$$

We now recall that an operator of the form $\theta h_{\overline{\zeta \zeta}}+\alpha h_{\zeta \zeta}+\beta h_{\overline{\zeta \zeta}}+\gamma \overline{h_{\zeta \zeta}}+\delta \overline{h_{\overline{\zeta \zeta}}}$ is elliptic and lies in the same homotopy class as the Laplacian if and only if

$$
|\theta+\alpha \lambda+\beta \bar{\lambda}|>|\gamma \bar{\lambda}+\delta \lambda|
$$

for every $\lambda \in \mathbb{C}$ with $|\lambda|=1$. For us the desired inequality reads, after a little simplification, as

$$
\begin{aligned}
& \left|\left(D-k^{2}\right) k^{4} a^{2} \bar{\lambda}+(D+1) b^{2} \lambda\right| \\
& \quad<\left|D^{2}\left(1-k^{2}\right)+2 D\left(1+k^{4}\right)+1-k^{6}-(D+1) k^{3} \bar{a} b \lambda+\left(k^{2}-D\right) k a \overline{b \lambda}\right| .
\end{aligned}
$$

In order to simplify this notation we introduce the new complex variable

$$
\xi=\bar{a} b \lambda, \quad|\xi|=1,
$$

and then we need to show, for $D>0$, that

$$
\begin{aligned}
& \left|\left(D-k^{2}\right) k^{4} \bar{\xi}+(D+1) \xi\right| \\
& \quad<\left|D^{2}\left(1-k^{2}\right)+2 D\left(1+k^{4}\right)+1-k^{6}-(D+1) k^{3} \xi+\left(k^{2}-D\right) k \bar{\xi}\right| .
\end{aligned}
$$

Before establishing this inequality we suggest that the reader checks the limiting and easier case $D=0$ (that is $K=\infty$ ) by verifying that

$$
\left|1-k^{6}-k^{3} \xi+k^{3} \bar{\xi}\right|=\left|\xi-k^{6} \bar{\xi}\right| .
$$

We now turn to establishing the ellipticity condition at (A.41). We write $\xi=x+i y$ for real $x$ and $y$, with $x^{2}+y^{2}=1$. We put this into the inequality and separate out the real and imaginary parts and then compute the square of the absolute values. We are thus asked to verify that

$$
\begin{aligned}
& {\left[\left(D-k^{2}\right) k^{4}+(D+1)\right]^{2} x^{2}+\left[\left(D-k^{2}\right) k^{4}-D-1\right]^{2} y^{2}} \\
& <\left[D^{2}\left(1-k^{2}\right)+2 D\left(1+k^{4}\right)+1-k^{6}-k D\left(1+k^{2}\right) x\right]^{2} \\
& \quad+\left[2 k^{3}+D k\left(k^{2}-1\right)\right]^{2} y^{2} .
\end{aligned}
$$


We next write $y^{2}=1-x^{2}$ to obtain a quadratic polynomial in $x$,

$$
\begin{aligned}
{\left[D^{2}(1-\right.} & \left.\left.k^{2}\right)+2 D\left(1+k^{4}\right)+1-k^{6}\right]^{2} \\
& -2 k D\left(1+k^{2}\right)\left[D^{2}\left(1-k^{2}\right)+2 D\left(1+k^{4}\right)+1-k^{6}\right] x \\
& +k^{2} D^{2}\left(1+k^{2}\right) x^{2}+\left[2 k^{3}+D k\left(k^{2}-1\right)\right]^{2} \\
& -\left[2 k^{3}+D k\left(k^{2}-1\right)\right]^{2} x^{2}-\left[\left(D-k^{2}\right) k^{4}+D+1\right]^{2} x^{2} \\
& -\left[\left(D-k^{2}\right) k^{4}-D-1\right]^{2}+\left[\left(D-k^{2}\right) k^{4}-D-1\right]^{2} x^{2}>0 .
\end{aligned}
$$

Next we group together the coefficients of $x^{2}$ and find that there sum is negative. That is, we see that

$$
\begin{array}{rl}
k^{2} D^{2}\left(1+k^{2}\right)-\left[2 k^{3}+D k\left(k^{2}-1\right)\right]^{2}- & {\left[\left(D-k^{2}\right) k^{4}+D+1\right]^{2}} \\
+ & {\left[\left(D-k^{2}\right) k^{4}-(D+1)\right]^{2}} \\
=-D^{2} k^{2}\left(1+k^{2}\right)\left(1+2 k^{2}\right)<0 & 0
\end{array}
$$

This then implies that the extreme case is when $x=1$ and $y=0$. Thus the inequality (A.41) reduces to showing that

$$
\left|D^{2}\left(1-k^{2}\right)+2 D\left(1+k^{4}\right)+1-k^{6}-k D\left(1+k^{2}\right)\right|>\left|\left(D-k^{2}\right) k^{4}+D+1\right| .
$$

That is,

$$
D^{2}\left(1-k^{2}\right)+D\left(2+2 k^{4}-k^{3}-k\right)>D\left(k^{4}+1\right) .
$$

Rearranging and simplifying, we see that this reduces to verifying that

$$
D^{2}\left(1-k^{2}\right)+D\left(1-k^{3}\right)(1-k)>0
$$

which, in view of the fact that $0 \leqslant k<1$, is clear.

\section{References}

1. B. Bojarski, 'On the first boundary value problem for elliptic systems of second order in the plane', Bull. Acad. Polon. Sci. Sér. Sci. Math. Astronom. Phys. 7 (1959) 565-570.

2. B. Bojarski and T. Iwaniec, 'Analytical foundations of the theory of quasiconformal mappings in $R^{n}$, Ann. Acad. Sci. Fenn. Ser. A I Math. 8 (1983) 257-324.

3. G. Choquet, 'Sur un type de transformation analytique généralisant la représentation conforme et définie au moyen de fonctions harmoniques', Bull. Sci. Math. 69 (1945) $156-165$.

4. B. Dacorogna, Direct methods in the calculus of variations, Applied Mathematical Sciences 78 (Springer, Berlin, 1989).

5. J. Douglas, 'Solution of the problem of Plateau', Trans. Amer. Math. Soc. 33 (1931) $231-321$.

6. P. Duren, Harmonic mappings between planar domains (Cambridge University Press, 2004).

7. L. C. Evans, 'Quasiconvexity and partial regularity in the calculus of variations', Arch. Rational Mech. Anal. 95 (1986) 227-252.

8. H. GRÖTzSCH, 'Über die Verzerrung bei schlichten nichtkonformen Abbildungen und über eine damit zusammenhängende Erweiterung des Picardschen Satzes', Ber. Verh. Sächs. Akad. Wiss. Leipzig 80 (1928) 503-507.

9. S. S. HABRE, 'The Fredholm alternative for second-order linear elliptic systems with VMO coefficients', Houston J. Math. 22 (1996) 417-433.

10. J. HADAmARD, Leçons sur la propagation des ondes et les équations de l'hydrodynamique (Hermann, Paris, 1903).

11. B. C. HALl, Lie groups, Lie algebras, and representations. An elementary introduction, Graduate Texts in Mathematics 222 (Springer, New York, 2003). 
12. S. Hencl, P. Koskela and J. Onninen, 'A note on extremal mappings of finite distortion', Math. Res. Lett. 12 (2005) 231-238.

13. T. IwANiEC, 'Green's function of multiply connected domain and Dirichlet problem for systems of second order in the plane', Function theoretic methods for partial differential equations, Darmstadt, 1976 (ed. V. E. Meister, N. Weck and W. L. Wendland), Lecture Notes in Mathematics 561 (Springer, Berlin, 1976) 261-276.

14. T. Iwaniec, P. Koskela and J. Onninen, 'Mappings of finite distortion: monotonicity and continuity', Invent. Math. 144 (2001) 507-531.

15. T. Iwaniec and A. Lutoborski, 'Integral estimates for null Lagrangians', Arch. Rational Mech. Anal. 125 (1993) 25-79.

16. T. Iwaniec and G. J. Martin, Geometric function theory and non-linear analysis, Oxford Mathematical Monographs (Oxford University Press, 2001).

17. J. Kauhanen, P. Koskela and J. Malý, 'Mappings of finite distortion: condition N', Michigan Math. J. 49 (2001) 169-181.

18. H. Kneser, 'Lösung der Aufgabe 41', Jahresber. Deutsch. Math.-Verein. 35 (1926) 123-124.

19. P. Koskela and J. Malý, 'Mappings of finite distortion: the zero set of the Jacobian', J. Eur. Math. Soc. 5 (2003) 95-105.

20. P. Koskela and J. Onninen, 'Mappings of finite distortion: capacity and modulus inequalities', J. reine angew Math. to appear.

21. M. Lavrentiev, 'Sur quelques problèmes du calcul des variations', Ann. Mat. Pura Appl. 4 (1926) $7-78$.

22. O. LeHto, Univalent functions and Teichmüller spaces (Springer, Berlin, 1987).

23. H. LEWY, 'On the non-vanishing of the Jacobian in certain one-to-one mappings', Bull. Amer. Math. Soc. 42 (1936) 689-692.

24. A. LyzzAIK, 'The modulus of the image annuli under univalent harmonic mappings and a conjecture of Nitsche', J. London Math. Soc. (2) 64 (2001) 369-384.

25. D. S. Mitrinović and P. M. Vasić, Analytic inequalities (Springer, New York, 1970).

26. C. B. Morrey, 'Quasiconvexity and the lower semicontinuity of multiple integrals', Pacific J. Math. 2 (1952) 25-53.

27. J. C. Nitsche, 'On the modulus of doubly-connected regions under harmonic mappings', Amer. Math. Monthly 69 (1962) 781-782.

28. M. PAvlović, 'Boundary correspondence under harmonic quasiconformal homeomorphisms of the unit disk', Ann. Acad. Sci. Fenn. Math. 27 (2002) 365-372.

29. T. Radó, 'Aufgabe 41', Jahresber. Deutsch. Math.-Verein. 35 (1926) 49.

30. Yu. G. Reshetnyak, Space mappings with bounded distortion, Translations of Mathematical Monographs 73 (American Mathematical Society, Providence, RI, 1989).

31. K. Streble, 'Extremal quasiconformal mappings', Results Math. 10 (1986) 168-210.

32. J. VÄıs̈̈LÄ, 'Two new characterizations for quasiconformality', Ann. Acad. Sci. Fenn. Ser. A I 362 (1965) 1-12.

33. G. Verchota, 'Harmonic homeomorphisms of the closed disk to itself need be in $W^{1, p}$, $1<p<2$, but not in $W^{1,2}$, Preprint, Syracuse University, 2004.

34. A. Weitsman, 'Univalent harmonic mappings of annuli and a conjecture of J. C. C. Nitsche', Israel J. Math. 124 (2001) 327-331.

\author{
K. Astala \\ Department of Mathematics \\ University of Helsinki \\ P.O. Box 4 \\ Helsinki \\ FIN-00014 \\ Finland \\ kari.astala@helsinki.fi \\ G. J. Martin \\ Institute of Information and \\ Mathematical Sciences \\ Massey University \\ Albany \\ Auckland \\ New Zealand \\ g.j.martin@massey.ac.nz
}

\author{
T. Iwaniec \\ Department of Mathematics \\ Syracuse University \\ Syracuse \\ NY 13244 \\ USA
}

tiwaniec@mailbox.syr.edu

\author{
J. Onninen \\ Department of Mathematics \\ University of Michigan \\ 525 East University \\ Ann Arbor \\ MI 48109 \\ USA \\ jonninen@umich.edu
}

